University of Louisville

ThinkIR: The University of Louisville's Institutional Repository

Electronic Theses and Dissertations

$5-2020$

\title{
Coparenting and school success: perspectives of fathers.
}

Shannon M. Mount
University of Louisville

Follow this and additional works at: https://ir.library.louisville.edu/etd

Part of the Social Work Commons

\section{Recommended Citation}

Mount, Shannon M., "Coparenting and school success: perspectives of fathers." (2020). Electronic Theses and Dissertations. Paper 3415.

https://doi.org/10.18297/etd/3415

This Doctoral Dissertation is brought to you for free and open access by ThinkIR: The University of Louisville's Institutional Repository. It has been accepted for inclusion in Electronic Theses and Dissertations by an authorized administrator of ThinkIR: The University of Louisville's Institutional Repository. This title appears here courtesy of the author, who has retained all other copyrights. For more information, please contact thinkir@louisville.edu. 


\title{
COPARENTING AND SCHOOL SUCCESS: PERSPECTIVES OF FATHERS
}

\author{
By \\ Shannon M. Mount \\ B.A., Indiana University, 1993 \\ M.S.W., Indiana University, 1995

\begin{abstract}
A Dissertation
for the Degree of

Doctor of Philosophy

in Social Work

Kent School of Social Work

University of Louisville

Louisville, Kentucky
\end{abstract} \\ Submitted to the Faculty of the \\ Raymond A. Kent School of Social Work of the University of Louisville \\ In Partial Fulfillment of the Requirements
}

May 2020 



\title{
COPARENTING AND SCHOOL SUCCESS: PERSPECTIVES OF FATHERS
}

By

\author{
Shannon M. Mount \\ B.A., Indiana University, 1993 \\ M.S.W., Indiana University, 1995 \\ A Dissertation Approved on
}

March 25, 2020

by the following Dissertation Committee:

\begin{tabular}{|c|}
\hline $\begin{array}{l}\text { Dissertation Director } \\
\text { Dr. Armon Perry }\end{array}$ \\
\hline Dr. Sunshine Rote \\
\hline Dr. Emma Sterrett-Hong \\
\hline Dr. Eli Karam \\
\hline
\end{tabular}

Dr. David Royse 


\section{ACKNOWLEDGMENTS}

I would like to thank my Dissertation Chair, Dr. Armon Perry, for providing me his professional expertise, guidance, patience and encouragement throughout this process. I would also like to thank Dr. Sunshine Rote for her feedback and direction through the analysis phase of this paper. Additionally, I would like to thank my family for allowing me the time away while I pursued this goal. Without the support of my husband, my parents, and my mother-in-law, I'm not sure I could have gotten this dissertation across the finish line. I want to thank them all for their assistance in this journey. Lastly, I would like to thank my four children, Kaleb, Isaak, Eliza and Rachael, for encouraging me and supporting me. I can only hope that accomplishing this task in the face of life challenges over the last few years can be an inspiration to them as they set out to accomplish their own dreams. 


\section{ABSTRACT \\ COPARENTING AND SCHOOL SUCCESS: PERSPECTIVES OF FATHERS \\ Shannon M. Mount}

March 25, 2020

Historically, measures of coparenting have been reported by mothers. As a result, fathers' perspectives and experiences have largely been excluded from this body of research. In response, this study relies on fathers own report of coparenting support. This study used a sample of 1255 biological fathers from the Fragile Families and Child Wellbeing Study (FFCWS) to explore fathers' reports of their coparenting support and how this is associated with relationship status with mother, time spent with child, and several school success variables including: internalizing and externalizing school behaviors and math and reading assessment scores at child age 9. Hypotheses included that higher coparenting reports would be positively associated with married and romantic

cohabitating fathers as well as fathers who spent more time with their child. Additionally, it was hypothesized that higher father coparenting reports would predict lower internalizing and externalizing behaviors and increased reading and math scores. While support was found for relationship status and time spent with child coparenting hypotheses, there was little support for the school variables to be significantly predicted by coparenting reports. Two control variables (race/ethnicity and poverty to income level) did yield consistent results for predicting school outcome variables. Future research should continue to focus on the equity of how coparenting is measured and reported and how the coparenting relationship is significant in healthy child development. 


\section{TABLE OF CONTENTS}

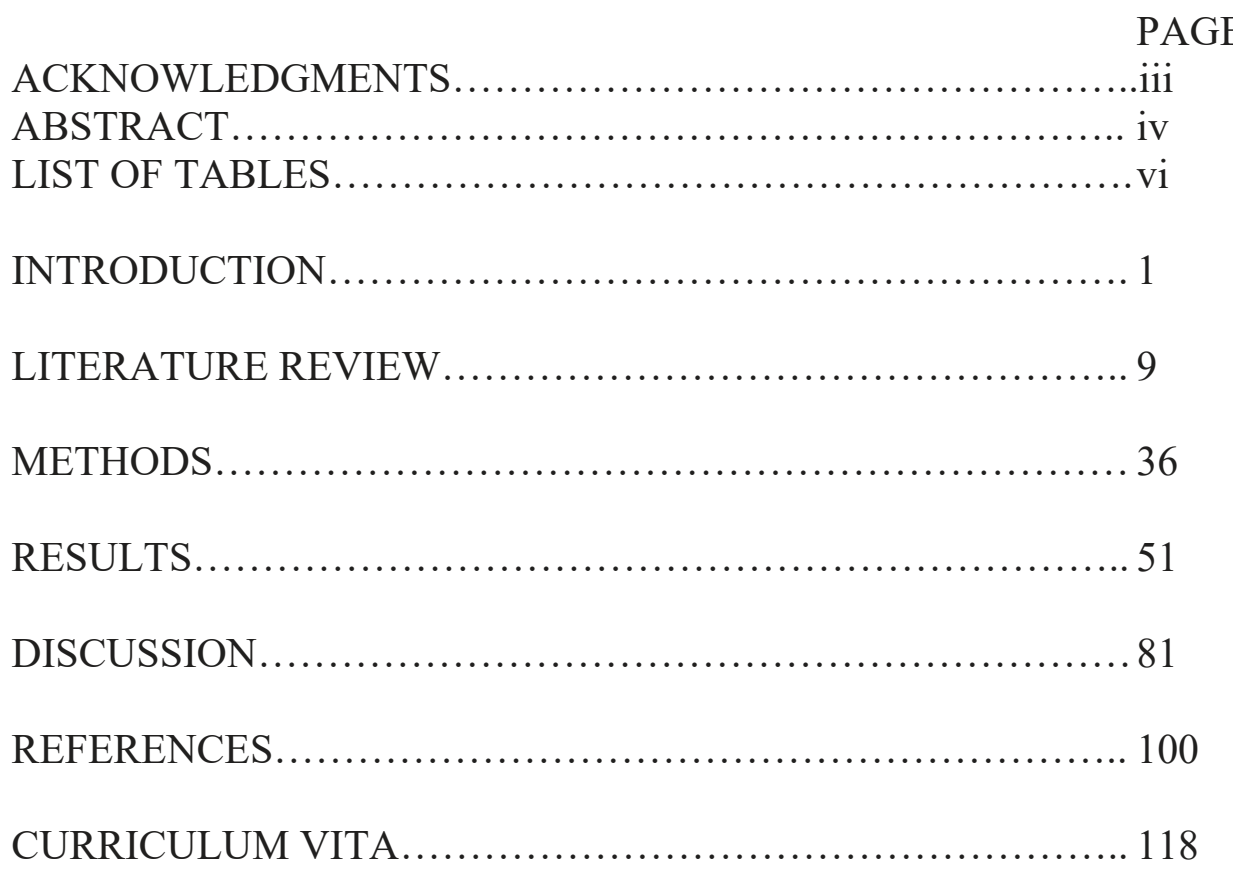




\section{LIST OF TABLES}

TABLE

PAGE

1. Table 1.1 Descriptive Statistics for Study Variables.......................... 53-54

2. Table 1.2 Bivariate Correlations........................................ 57

3. Table 1.3 Research Question 1 ANOVA with Tukey post hoc results............61

4. Table 1.4 Research Question 1 Multinomial Logistic Regression results.........62

5. Table 2.1 Research Question 2 ANOVA with Tukey post hoc results............66

6. Table 2.2 Research Question 2 Multinomial Logistic Regression results.........67

7. Table 3.1 Summary of OLS Regression Analysis for Variables Predicting Child's Reading Scores at age

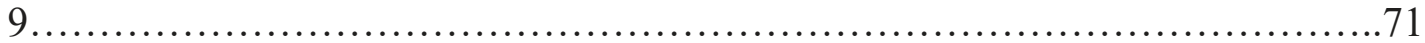

8. Table 3.2 Summary of OLS Regression Analysis for Variables Predicting Child's Math

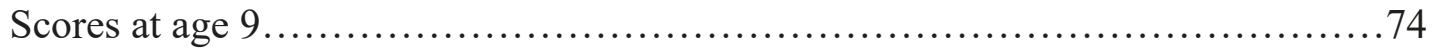

9. Table 3.3 Summary of Binary Logistic Regression Analysis for Variables Predicting

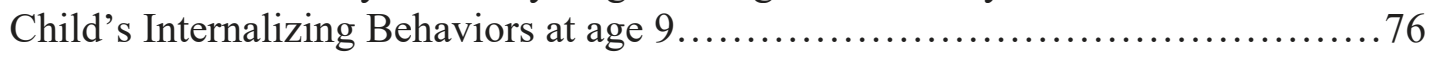

10. Table 3.4 Summary of Binary Logistic Regression Analysis for Variables Predicting

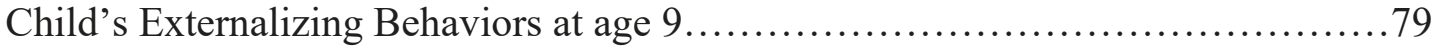




\section{CHAPTER 1: INTRODUCTION AND STATEMENT OF PROBLEM}

Families are an important foundation for many parts of a child's life. Family systems theory provides a mechanism for examining the family system and the effects this system has on child development (Bronfenbrenner, 1990). One important executive subsystem in the family is the coparenting system (Minuchin, 1974). The coparenting subsystem is a distinct and complex part of the parents' relationship because of how it affects the child (Feinberg, 2003; McHale, Kuersten-Hogan, \& Rao, 2004; McHale \& Lindahl, 2011). When a child enters the formal schooling years, the foundation the family has built for the child can predict a child's success. Recently researchers have begun to focus on how the coparenting mechanism of the family system relates specifically to school outcomes, such as academic and social skills functioning. (Belsky \& Fearon, 2004; Cabrera, Scott, Fagan, Steward-Streng, \& Chien, 2012). Preliminary results suggest that coparenting conflict may interfere with a child's development and affect the child school success with links to lower academic skills and higher incidences of poor social skills. This has led researchers to investigate spillover effects of coparenting difficulties linking to higher rates of depression and antisocial behaviors in children (Feinberg, 2003; Feinberg, Kan \& Hetherington, 2007).

Given the impact that the coparenting relationship plays on children's educational outcomes, it is important that social workers better understand the factors that contribute to the development of a positive coparenting relationship. A review of the literature reveals that the Feinberg (2003) coparenting model has wide utility and applicability in 
its ability to explain coparenting relationship dynamics. In this model, four coparenting components can be used to explore the complex coparenting relationship. Figure 1 (below) shows a visual representation of Feinberg's Model of Coparenting Components. The proposed study examines the coparenting relationship in diverse family systems based on Feinberg's Model of Coparenting Components and the association with children's educational outcomes. The following sections describe key terms that will be used in the study in an effort to provide clarity.

\section{Key Terms: Family Diversity and the "Fragile Family"}

A report by the Pew Research Center details the changes in family structure and concludes that there is no one dominant form of family structure in the U.S. anymore. In 1960, the dominant form of family structure was two married parents in their first marriage living with children. This accounted for $73 \%$ of all children (Pew Research Center, 2015). Four in every ten births in the U.S. are to women who are single or cohabitating. Less than half (46\%) of children are being raised in what used to be defined as a traditional family, two biological parents in their first marriage (Pew Research Center, 2015).

Another component to understanding changes in family structure is the cohabitating but never-married population that is parenting children under 18. The number of couples cohabitating, but not married, has steadily risen from 1.2 million in 1996 to over 3.3 million in 2015 (Child Trends Databank, 2015). Compared to married couples, cohabitating couples as a group tend to have some common characteristics including being younger, having less education, having lower income, and having less secure employment (Child Trends Databank, 2015). 
Since the 1960s, there has been a shift in family composition away from the traditional, married, two parent family. This change in family demographics has called attention to the population commonly referred to as "fragile families." The term fragile family is used to refer to low income or unmarried parents and their children. The use of this terminology is meant to draw attention to the fact that (1) they are indeed families, but (2) these families are at higher risk for adverse life conditions such as poverty and family dissolution (McLanahan, Garfinkel, Reichman, Teitler, Carlson, \& Audigier, 2003).

\section{Key Terms: Coparenting}

McHale, Lauretti, Talbot and Pouquette (2002) describe coparenting as "an enterprise undertaken by two or more adults working together to raise a child for whom they share responsibility" (p.127). This definition speaks of working together as a team and lends itself well to many diverse family configurations. While the coparenting unit has traditionally been thought of as the married or post-divorce couple with children, the McHale et al. (2002) definition allows for never-married coparenting populations, whether they are cohabitating or noncohabitating, to be included.

\section{Key Term: Interparental Conflict versus Coparenting Conflict}

It is important to note that the terms "interparental conflict" and "coparenting conflict" are not interchangeable. In family research, interparental conflict generally refers to the all-encompassing conflict that can take place in the adult relationship between parents. This can include conflict about such things as the romantic relationship between two parents, the communication issues between parents, and even the financial conflict between parents. One sub-set of interparental conflict is coparenting conflict which refers 
to the conflict that comes when parents disagree on how to tackle parenting decisions together as a team.

\section{Key Terms: Relationship Quality}

Relationship quality refers to the romantic relationship between the mother and father in the family unit. In the research literature, relationship quality is generally studied where the mother and father are still romantically involved but could be in a variety of relationship statuses including married, nonmarried cohabitating, or nonmarried noncohabitating, yet still together as a romantic couple. The quality of the couple's romantic relationship has been found in previous studies to be an antecedent to the quality of the coparenting relationship (Goldberg \& Carlson, 2015; Carlson \& Hognas, 2011; Kamp Dush, Kotila \& Schoppe-Sullivan, 2011). However, while relationship quality and coparenting are linked, each are distinct family dynamics (McHale, Lauretti, Talkbot, \& Pouquette, 2002). This study will be focusing specifically on the coparenting part of the parenting relationship.

\section{Key Term: Middle Childhood}

While there is a lack of congruence on the exact boundary of middle childhood, it is generally referring to a child between the ages of 6-10 in Western culture (Hutchinson, 2015). This stage is marked by continual physical development, cognitive, emotional, and social development. It is often referred to as a "school-age" developmental phase as many cultures turn to formal education during these years (Hutchison, 2015).

\section{Study Parameters}

It is important to note that researchers in the field of family functioning have explored other diverse coparenting configurations outside the confines of a child's 
biological parents including extended diverse kinships systems in different cultures (e.g. Jones \& Lindahl, 2011; Gonzalez, Jones, \& Parent, 2014); same sex parents (e.g. Patterson \& Farr, 2011; Farr \& Patterson, 2013); and coparenting in foster family situations (e.g. Linares, Rhodes, Montalto, 2010; Montalto \& Linares, 2011). However, the focus of this study is on biological parents who are coparenting in a variety of union statuses including married cohabiting, divorced, nonmarried cohabitating, and nonmarried noncohabitating. Based on previous family structure and functioning research, there are several reasons to examine the experiences of biological fathers.

Firstly, one intent of this dissertation is to fill a research gap by amplifying the voices of fathers who were in noncohabitating relationships with the focal child's mother. Before 1998, when the Fragile Family and Child Wellbeing study began, the most complete study with unmarried fathers was the National Survey of Family and Households. However, a major limitation of this study is that they missed a sizeable number of biological fathers who lived apart from their children (McLanahan \& Carlson, 2004). Researchers estimated that $40 \%$ of nonresident biological fathers were missing from the National Survey on Family and Households either because they were not interviewed at all or because they were not identified as fathers (Garfinkel, McLanahan, \& Hanson, 1998).

Secondly, shifts in relationships and families have also contributed to the increase in individuals having children with more than one partner, referred to as "multiple partner fertility" or "multipartnered fertility" in the research literature (Carlson \& Furstenberg, 2006). With each transition of a father or father figure in and out of the child's life, the child is put at more risk for externalizing behaviors (Bronte-Tinkew, Horowitz, \& Scott, 
2009; Carlson \& Furstenberg, 2006). Additionally, when a mother has children with another partner, the biological father of previous child/ren may get pushed out of coparenting relationship by the new "social" father (Roopnarine, 2004). Martin, Ryan, Riina, and Brooks-Gunn (2017) found that when biological fathers were in the household there was a large increase in coparenting quality and consequently, when a social father entered the family it consistently interfered with the biological father's coparenting relationship.

Lastly and perhaps most importantly, children benefit when their biological fathers are involved. The biological father-child attachment relationship has been linked to the coparenting relationship. Brown, Schoppe-Sullivan, Mangelsdorf and Neff (2010) found that supportive coparenting resulted in greater infant-father attachment but not greater infant-mother attachment.

\section{Theoretical Considerations: Feinberg's Model of Coparenting Components}

Feinberg (2003) proposes a model which uses four distinct factors to define the coparenting relationship. The purpose of the Feinberg model was to show the unique, complex, interactive and overlapping dynamics of the coparenting relationship. These components are: (1) childrearing agreement/disagreement (Ex. agreement on how to discipline after a child's misbehavior); (2) division of child-related duties, tasks, and responsibilities (Ex. who gets the child up, fed and dressed in the morning); (3) joint management of family interactions (Ex. being a coparenting team rather than trying to pull the child to one parent's side); and (4) coparenting support/undermining (Ex. respecting the other parent's decisions and upholding these with the child). The 
components of this model will be used to explore and assess the coparenting relationship.

Figure 1 (below) provides a visual representation of the model.

\section{Research Questions}

The aim of this research is to examine the coparenting relationship in diverse family systems using Feinberg's Model of Coparenting Components (Feinberg, 2003) and the subsequent effects the coparenting relationship has on the child's educational outcomes at age 9 .

Research Questions include:

1a. Are there differences in father's perceptions of coparenting by relationship status (with child's mother)?

1b. Are there significant differences when controlling for background factors (education, poverty level, age, race/ethnicity)?

2a. Are there differences in father's perceptions of coparenting by time spent with the child?

$2 \mathrm{~b}$. Are there significant differences when controlling for background factors (education, poverty level, age, race/ethnicity)?

3a. Are father's perceptions of coparenting associated with the focal child's school success (academic and behavioral measures) at age 9?

$3 \mathrm{~b}$. Are there significant differences when controlling for background factors (education, poverty level, age, race/ethnicity)? 


\section{Figure 1: Feinberg's Model of Coparenting Component}

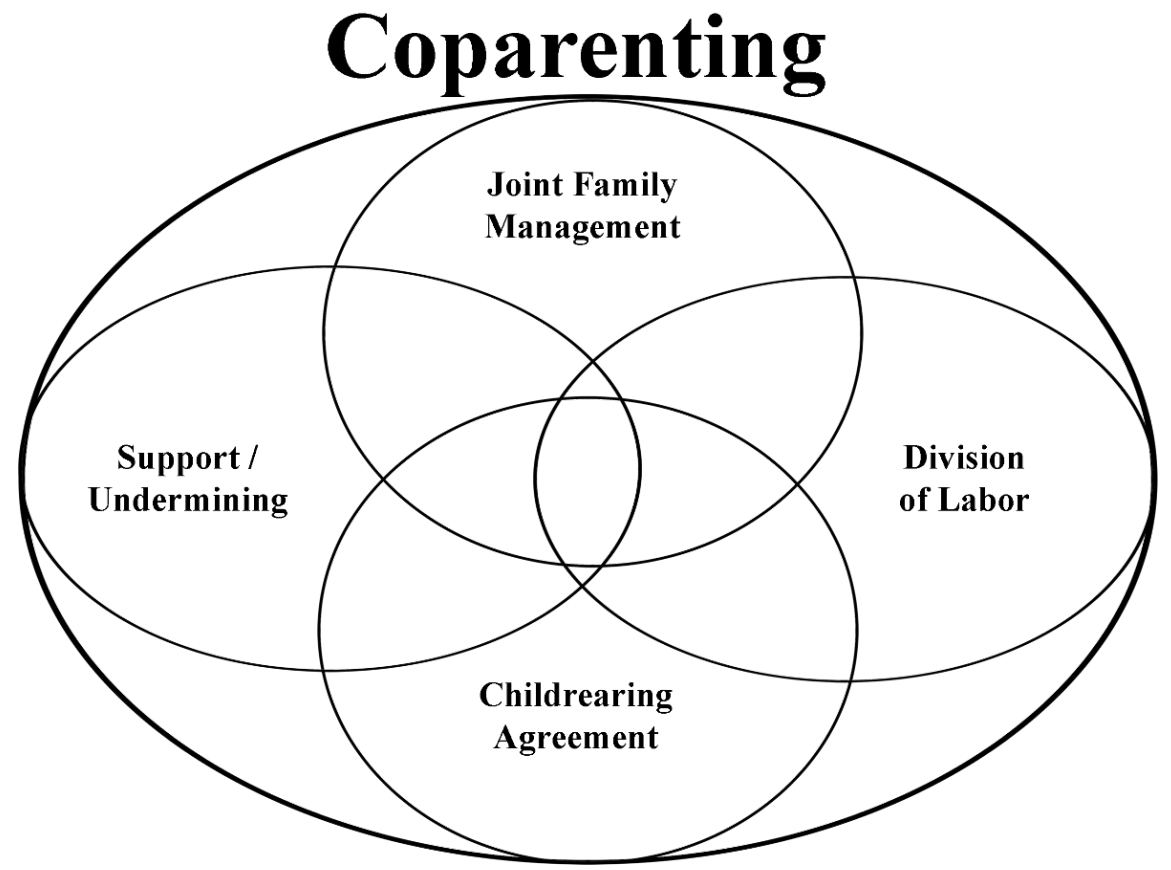

Feinburg's Model of Coparenting Components 


\section{CHAPTER 2: LITERATURE REVIEW}

This chapter provides the reader with a review of the literature including the theories of ecological, family, and coparenting systems, as well as the background on Feinberg's Model of Coparenting. There is also a chronological discussion on the research involving coparenting with a historical perspective on divorced coparenting, culminating in current research on coparenting in "fragile families." Next, the most current research regarding coparenting and educational outcomes is presented. Lastly, research gaps in the most recent literature are discussed to provide focus for the current study.

\section{Theoretical Background: Ecological Systems \& Family Systems Theory}

Systems theory is based on the premise that the whole is more than the sum of its parts. In the study of families, this premise implies that the family system is more than just the sum of the various individuals that make up its membership. Families are complex, forming an interdependent system where the decisions of the individual or a dyad can affect the entire family.

Social Work has a rich history of studying families as systems. Ecological systems theory (also referred to as the bioecological system theory) first gained attention with Urie Bronfenbrenner's work entitled The Ecology of Human Development (1979). In this work, Bronfenbrenner suggested that families are nested systems of interaction and proposed four levels of systems for analysis. These four levels are as follows: (1) the 
microsystem, which consists of the individual and the interpersonal relationships closest to the individual; (2) the mesosystem, which consists of the interactions of two or more of the microsystems; (3) the exosystem, which embraces the larger societal interactions; and (4) the macrosystem, which consists of the cultural context that informs the generational patterns of behaviors (Bronfenbrenner, 1979). Bronfenbrenner added a fifth level called the chronosystem which consists of the patterns of behaviors over time and/or life span (1979).

Since the 1950s, social scientists have acknowledged the family system as a primary influence across the lifespan. This was a theoretical and clinical shift from focusing primarily on the psychopathology of an individual to focusing on how the individual interacts within the systems in which they live. Cowan (2002) summarizes family systems theories as sharing six key assumptions: (1) the whole is greater than the sum of its parts; (2) the family system is made up of interconnected subsystems (parents, parent-child, siblings); (3) since the subsystems are interconnected, any changes in individuals and/or relationships will subsequently affect all the other individuals and/or relationships in the family system; (4) causality is multidirectional rather than linear (parents affecting children as well as children affecting parents); (5) family systems (like many other systems) have self-balancing and self-regulating mechanisms; and (6) interventions in family systems help to understand how the systems function.

\section{Theoretical Background: The Coparent Executive Sub-system}

McHale and Lindahl (2011) assert that studying the coparenting relationship in a systems framework can trace back to Salvador Minuchin's Families and Family Therapy (1974). In this work, Minuchin notes that the coparenting system is the executive 
subsystem of the family system. Although Minuchin refers specifically to the traditional two-parent system, the coparenting system has been expanded to include diverse coparenting systems such as cohabitating coparents, non-marital coparents such as mother-grandparent coparents, and even foster families (McHale \& Lindahl, 2011). Minuchin (1974) claims that for children to thrive both behaviorally and emotionally, they need an executive coparenting system that operates with joint authority, works as a team, shares roles, and operates with distinct boundaries to make the family a unit. Historically this coparenting system was thought of as dyadic (parent-parent or parent-child), with specific attention to the mother-child dyad. However, Patricia Minuchin (1985), daughter of Salvador Minuchin, wrote an article that pushed the limits of the dyadic approach to another level of thinking of family systems. She proposed expanding family systems thinking into a triadic approach where each member of the family was simultaneously involved with the other two members of the triad (e.g. parent to parent-child; child to parent-parent). This thinking expanded the complexity of family systems research. Despite her argument for triadic systems, however, most interventions are still conducted with the mother-child dyad either in a home-based or office-based setting (McHale, 2007). Even with the increased focus on the importance of fathers in the family systems, there are very few fatherhood initiatives that engage the entire coparental-child family system.

Within the family systems framework, there is something known as the spillover effect. Researchers such as Crouter (1984) first started studying the spillover effect within the dynamics from work to home subsystems. Spillover occurs when tension in one part of a system (e.g. parent-parent dyad) causes a direct impact (either positive or 
negative) on another system (e.g. parent-child dyad). These spillover effects can also be bi-directional where marital discord can cause parental discord which in turn compounds marital discord (Saxbe, Rodriguez, \& Margolin, 2013). In a meta-analysis of 68 studies, Erel and Burman (1995) found a clear relationship between marital spillover effects and negative marital relationship consequences on the parent-child relationship.

\section{Theoretical Background: Feinberg's Model of Coparenting Components}

The coparenting relationship has been linked to child emotional and behavioral problems (e.g. Goldberg \& Carlson, 2014; Fomby \& Cherlin, 2007; Cummings \& Davies, 2002). Early researchers in the field of family functioning found preliminary evidence that coparenting dynamics were linked to a child's emotional security and thus affected other areas of the child's life (Cummings \& Davies, 1994; Cummings, Davies \& Simpson, 1994). Because the coparenting relationship was found to be linked to child development in such an impactful way, Feinberg felt that it was important to break down the general term "coparenting" into the main components that make up the coparenting relationship (Feinberg, 2003).

Feinberg $(2002,2003)$ set out to propose a model of coparenting that would focus on the different components that make up the coparenting relationship (see Figure 1). In attempting to study the family system, he wanted to provide domain specificity to the coparenting part of the interparental subsystem. In doing so, he focuses on the overlapping and shared responsibilities that come when two people work together to parent a child. He specifically separates out those parts of the dyadic couple relationship (sometimes referred to as the romantic or marital relationship) which includes the romantic, sexual, financial, legal, emotional and companionable parts of the relationship. 
Whereas a couple's relationship is focused on the self and/or the couple's wellbeing, the coparenting relationship is built on the mutual concern for the wellbeing of the child/ren. Feinberg builds on the work of other family researchers (e.g. McHale, 1995; Margolin, Gordis, \& John, 2001) to portray coparenting as a four-component model. These four components include: (1) childrearing agreement/ disagreement; (2) division of childrelated duties, tasks, and responsibilities; (3) joint management of family interactions; and (4) coparenting support/undermining. Each of the four components are separate yet overlapping, indicating that an unhealthy imbalance in one could potentially affect another.

Childrearing agreement/disagreement refers to the level of agreement that coparents have on a variety of childrearing issues including agreed upon values, the child's developmental behavioral expectations, meeting and managing the child's emotional needs, discipline, educational standards, safety, and peer relationships to name a few. Like the other components, childrearing disagreement by itself will not completely explain poor child outcomes. For example, parents can "agree to disagree" on a parenting practice as long as this does not significantly imbalance the other components of the coparenting relationship such as mutual support and joint family management processes. However, if there is acute and chronic disagreement on childrearing practices, then this one component could be enough to upset the entire coparenting relationship (e.g. Grych \& Fincham, 1990; Van Egeren, 2003).

The second component is division of labor. This includes division of daily parenting responsibilities and tasks of direct childcare. It also includes those tasks and responsibilities such as the educational, financial, legal, and medical needs associated 
with the indirect care of the child. This division varies across households, but the most important piece to this component is that those involved in the coparent relationship agree to the division of labor and describe it as fair.

The third component is support-undermining. This is a measure of the parent's supportiveness to the other. This includes affirming the other parent's competency as a parent; acknowledging and respecting the other parent's contributions; and upholding the other parent's decisions and authority in the family system.

The final component is joint family management. This fourth component is separated into: interparental conflict, coalitions, and balance. Interparental conflict is described as parents' responsibility for controlling their own behaviors and communicating with each other. Previous researchers have found that children exposed to frequent, unresolved, and/or hostile interparental conflict can result in poor child adjustment and development (e.g. Grych \& Fincham,1990). Feinberg defines coalitions as a parent's behaviors and attitudes that set boundaries on how they choose to parent. This is the extent in which one parent will decide to engage or exclude the other parent. Triangulation can occur when a parent is attempting to pull the child over to his/her way of rationalizing a behavior or decision. This triangulation can be overt or covert with the child being pulled into a parent-child alliance rather than a parent-parent alliance. Balance is the third part of joint family management. It is described as the balance in the parents' interactions with the child. This is not so much a measure of equal time, but an attempt at balancing the coparenting relationship. A balanced coparenting relationship would be one where each parent encourages the involvement of the other parent with the child in an effort to promote healthy child development. 
A Google Scholar search of Feinberg's original 2003 article reveals that it has been cited in more than 530 subsequent works in family research since its publication. Researchers have used Feinberg's coparenting model as a basis to study coparenting function in areas such as the transition to parenting (e.g. Kuersten-Hogan, 2017); coparenting scale development (e.g. McDaniel, Teti, \& Feinberg, 2017); coparenting in divorced families (e.g. Petren, Ferraro, Davis, \& Pasley, 2017); coparenting in adolescent/teen parents (e.g. Sznitman, Van Petegem, \& Zimmermann, 2018); the role of coparenting in maternal gatekeeping (e.g. Schoppe-Sullivan, Altenburger, Lee, Bower, \& Kamp Dush, 2015); coparenting and father engagement (e.g. Fagan \& Cabrera, 2012); and the expansion of coparenting theory and further research (McHale, Kuersten-Hogan, \& Rao, 2004).

\section{Family Systems Theory}

Family Systems Theory is one of the ways that researchers strive to understand all that happens within the family environment. While this perspective cannot be attributed to any one person, researchers using the family systems model generally agree on a common set of criteria (e.g. Cox \& Paley, 1997, Minuchin, 1985). These common criteria consist of understanding the following about families: (1) that families are viewed as an organized system made up of interdependent subsystems, (2) that a family's subsystems can be dyads, triads or even larger units, (3) that three key components are important when understanding family interactions. These three key components are wholism (also referred to as holism), interdependence, and boundaries (Grych, Oxtoby, Lynn, 2013).

Wholism refers to how the whole family system cannot be simply reduced to its parts or subsystems. The interconnected relationships of the subsystems are so important 
that the subsystems cannot be understood as a detached entity (Grych, Raynor, \& Fosco, 2004). Interdependency is the concept that the individuals, the dyads and triad subsystems, and their relationships to each other mutually influence each other. Each part of the family system affects other parts of the family system. Finally, boundaries in family systems refers to the how members of the systems are defined and how they interact. For instance, family system boundaries can range from enmeshed to disengaged. However, researchers have observed that healthy family systems are able to be flexible with boundaries rather than rigidly on one end of the continuum or the other (Grych, Oxtoby, Lynn, 2013).

Boyd-Franklin (2003) in working with families refers to the multisystem levels in which families can be viewed. She defines these levels as: (1) individual, (2) family subsystems, (3) family household, (4) extended family, (5) non-blood kin and friends, (6) church and community resources, and (7) social service agencies and other outside systems. Interventions with any of these systems can affect the family. This understanding of a multisystem ecological approach is key to understanding how the various levels might influence another level. This approach allows for cultural explanations and differences. For instance, this view might help explain how cultures in which intergenerational families who live together might be influenced in a different way from cultures where intergenerational living arrangements are not as common.

\section{Emotional Security Theory}

Davies and Cummings (1994) developed the Emotional Security Theory (EST).

In this theoretical framework, the authors propose that for the sake of healthy child mental health development parents need to form a secure foundation for healthy 
emotional development. Parents do this by providing comfort, protection, and family stability. Unhealthy interparental conflict, such as unresolved conflict, passive-aggressive disengagement, verbal aggression, and physical aggression, thus diminishes or threatens the child's sense of emotional security and impedes their goal of safety within the family.

Davies and Cummings (1994) found that when children are consistently exposed to a toxic conflictual environment between parents, their responses fall into three processes: (1) emotional reactivity (intense dysregulated distress when exposed to parents' conflicts), (2) regulation of exposure (displayed by heightened avoidance or involvement in parent's conflict), and (3) internal representations (working mental models that start to define expectations of self and relationships) (Davies \& Cummings, 1994). The third process is especially harmful to a child's development. As the child attempts and then fails to find emotional security within the family system, he/she starts to form internal representations of how parental relationships work. Davies and Cummings theorize these responses increases the child's chance at future psychopathology including internalizing problems (e.g. anxiety, depression), externalizing problems (e.g. conduct problems, aggression toward others), social impairments (peer difficulties) and school maladjustment (academic difficulties, poor peer relations at school) (Davies \& Martin, 2013; Grych \& Fincham, 2001).

In the years since Davies and Cummings first proposed and initially tested the theory, researchers have used it many times to test emotional security as a mediator between interparental conflict and poor child outcomes (e.g. Davies \& Martin, 2013; Sturge-Apple, Davies, Winter, Cummings, \& Schermerhorn, 2008; Cummings, Schermerhorn, Davies, Goeke-Morey, \& Cummings, 2006). 


\section{Family Stress Model}

Conger and colleagues $(1992,1993)$ first proposed the Family Stress Model when working with a large sample of rural families in the 1990s. They proposed that economic hardship leads to economic stress which in turn leads to parents being in an emotionally distressed state which in turn spills over into their interparental relationship. This conflict between parents makes the parents less available to their children leading to child adjustment problems including behavioral difficulties and impaired functioning (Donnellan, Martin, Conger, \& Conger, 2013).

In the first research study in 1992, the researchers found that evidence to support that economic stress was indirectly linked to adolescent boy behaviors and prosocial adjustment (Conger, Conger, Elder, Lorenze, Simons \& Whitbeck, 1992). This study focused on boys only. Their next study focused on adolescent girls. The girls' study replicated the findings of the boys' study (Conger, Conger, Elder, Lorenz, Simons \& Whitbeck, 1993).

Since those original studies, the work has been replicated with many diverse populations and support found for the Family Stress Model (FSM). Brief summaries of the results include: (1) support for FSM with low income/mostly single mother minority families (Mistry, Vandewater, Huston, \& McLoyd, 2002; Mistry, Lowe, Benner \& Chien, 2008), (2) support for FSM in African American families in both urban and rural settings (Conger, Wallace, Sun, Simons, McLoyd, \& Brody, 2002), (3) partial support for FSM in European American, Mexican American, and Chinese American families in California. Results showed there were parental gender (mother/father) differences in how economic stress affected parenting practices. Researchers in these studies suggested that culture 
influenced the gender differences. (Behnke, MacDermid, Coltrane, Parke, Duffy, \& Widaman, 2008; Benner \& Kim, 2010), and (4) support for FSM in families living outside the U.S. (Zhang, 2014; Leinonen, Solantus, \& Punamäki 2002).

\section{Review of the Literature}

\section{Coparenting in Divorced Families: A Historical Perspective}

The bulk of the early research literature concerning coparenting quality and its effects on the child resides in the study of divorced middle-class parents (e.g. Ahrons, 1981; Emery, 1982; Wallerstein \& Kelly, 1980). Ahrons (1981) conducted early qualitative research with divorced parents and found that the level of interaction between parents seemed to be an indicator of the quality of the coparenting relationship. This was the first time the coparenting relationship was categorized in terms of low, medium, and high-quality interactions. Emery (1982) is often cited as a publication that provided evidence that coparents' level of conflict was more important than the separation/divorce itself. In his article, he reviewed the few studies previously published to surmise that coparental conflict might be a key to explaining children's behavioral and emotional difficulties as opposed to the separation or divorce itself (Emery, 1982). It was this time in history that researchers started to question if "staying together for the child's sake" made sense, especially if a separation/divorce might alleviate a child's exposure to coparental conflict. In a review of the literature from 1980 to the late 1990's, there was sizable evidence to support that the conflict between parents was a better predictor of a child's behavioral and emotional wellbeing than the actual family structure (intact marriage or separated/divorced) (Buchanan \& Heiges, 2001). 
Amato $(2000,2001)$ reviewed literature since the 1990s on the subject of child outcomes from divorced-parent homes as compared to married-parent homes. He examined the effect sizes of research studies and found that children of divorced parents have poorer outcomes in academic performance, behavioral and emotional issues, interpersonal relationships, and self-esteem. He found that the gender of the child did not make a significant difference in the findings. He also found that the success of the child's adjustment to the divorce/separation was largely dependent on four factors: (1) the level of coparental conflict before and after the separation, (2) the quality of parenting, (3) changes in the child's economic stability, and (4) transitional stresses such as moving houses or having the move to another school (2001).

Emery partnered with Kelly in 2003 to update his original review. They summarized the effects of divorce on children and found that the absence or presence of stressors in a child's life affects the child's emotional behavioral outcomes. These stressors were (1) nature surrounding the initial parental separation, (2) parent adjustment and access to resources (such as economic and therapeutic), (3) level of parental conflict or cooperation, (4) re-partnering of one or both parents, (5) economic stability, and (6) the child's own individual resources (such as coping skills and emotional regulation) (Kelly \& Emery, 2003). This research also added support to Amato's $(2000,2001)$ previous body of work in that children from divorced families were found to have significantly more behavioral (both externalizing and internalizing), social, and academic problems when the group was compared to children in continuously married families (Kelly \& Emery, 2003). 
In their book summarizing three longitudinal studies, Hetherington and Kelly (2002) found that $25 \%$ of youths from divorced families had serious social, emotional or psychological problems when compared to only $10 \%$ from non-divorced families. They also concluded that the transition period in which the marriage is ending is an especially stressful period for children who are often dealing with multiple transitional stressors including a parent leaving the home, possible moves, changes in economic resources available, etc. Finally, they found that children want contact with both parents and that children can thrive in any custodial situation (mother, father or joint) as long as the parents are loving, firm, consistent in their parenting style, and also keep the coparental conflict low (Hetherington \& Kelly, 2002).

In 2010, Amato compiled another literature review to add to his prior works. He concluded that studies continue to show that there are differences between wellbeing measures with children from divorced families when compared to children of alwaysmarried parents. He also pointed out that the last decade of studies had started to focus on the quality of the relationship prior to the separation/divorce. In relationships that demonstrated a high amount of family dysfunction, the separation/divorce resulted in improvements in child wellbeing (Amato, 2010). His review also found studies that were starting to identify the number of transitions that the family system experiences as a possible predictor of child outcomes as opposed to a single event (the divorce of parents).

When a marriage relationship ends and children are part of the family system, the remaining relationship hopefully leaves room for a continued coparenting relationship which focuses on the health and wellbeing of the children. In a meta-analysis studying the association between coparenting and child behavioral and emotional outcomes, 
Teubert and Pinquart (2010) found that the way in which parents work together in a coparenting relationship was significantly related to the child's psychological adjustment. Buchanan and Heiges (2001) put separated/divorcing parents into three categories as follows: (1) active coparenting as defined by interacting directly with each other, (2) passive coparenting as defined by avoiding contact but being available to parent when it is their parenting time, and (3) no coparenting as defined when one parent is absent and not involved in the parenting relationship.

\section{Coparenting and the Couple Relationship}

The coparenting relationship and the couple relationship have been studied for their unique relationship properties that each bring to the overall concept of parenting. As stated before, the coparenting relationship relates to the ways that parents related to each other in the role of parent (Feinberg, 2003). Whereas the couple relationship pertains to "the romantic, sexual, companionate, emotional, financial, and legal aspects of the adult relationship that do not pertain to childrearing" (Feinberg, 2003, p.96). Research has found evidence to support that a healthy coparenting relationship can exist even if the couple relationship is not so healthy. However, it is not as likely that the couple relationship will be healthy if the coparenting relationship is unhealthy (Margolin, Gordis, \& John, 2001). Feinberg, Kan and Hetherington (2007) found evidence to support that the coparenting relationship was of greater importance to parenting when compared to the couple relationship. Given these findings between the couple relationship and the coparenting relationship, family researchers have generally agreed that the quality of the coparenting relationship is more likely than the couple relationship to have a causal 
relationship with individual child difficulties (Feinberg \& Sakuma, 2011; Margolin, Gordis \& John, 2001).

\section{Contemporary Families}

As stated previously, the U.S. family has changed in the last 50-60 years. Family researchers have come to use the term "family complexity" to refer to the observed changes in family composition since the World War II era (e.g. Cherlin \& Seltzer, 2014; Sawhill, 2014; Brown, Manning \& Stykes, 2015). Some ways in which families are more complex include (1) the higher percentages of children being born outside of marriage (nonmarital fertility), (2) the higher number of single mother households, (3) the higher number of parents choosing to cohabitate instead of legally marrying (nonmarital cohabitation), (4) the increasing number of children living with grandparents (intergenerational families), (5) the increasing number of children who are living with a step or half-sibling (multipartnered fertility, social father), (6) the emergence of new relationship patterns such as "living apart together" (LAT/couples who live apart yet define themselves as a couple), and (7) the increasing number of family transitions a child experiences (entrances and/or exits of relationships in a child's life). Meyer and Carlson (2014) sum it up this way, "economically disadvantaged men and women are more likely to have births outside of marriage, more likely to cohabit, more likely to have multiple unions, and more likely to have had children with more than one partner" (p. 260). From the child's perspective, these family changes and growing family complexity have been found to compromise well-being most notably in externalizing behaviors

including behavior problems and delinquency (Bronte-Tinkew, Horowitz, \& Scott, 2009; Carlson \& Furstenberg, 2006: Fomby \& Cherlin, 2007). 
In a special issue of The Annals of the American Academy dedicated to family complexity, Sawhill (2014) notes that socioeconomic status is closely related to the issue of family complexity. Brown, Manning and Stykes (2015) found that family complexity was independently related to economic disadvantage. Children are more than twice as likely to be part of a complex family if neither of the parents have a college degree (Manning, Brown, \& Stykes, 2014).

In fact, there is a gap continuing to grow between the economic groups of children living with both biological parents and children living in more complex family configurations. Furstenberg (2014) refers to this growing gap as a two-tiered family system where the practice of what a family consists of has become very different between the higher and lower socioeconomic classes, mimicking the gap in wages in the US. The top tier is made up of more well-educated and affluent partners who are entering marriage late in life most often after careers have been established. For this group, marriage is happening only after a long period of cohabitation. This group is also waiting longer to have children. In the second tier, couples are less educated and are most likely to cohabitate instead of legally marrying. Couples enter into this cohabiting arrangement shortly before or just after conception. Hopes of marriage are talked about after the birth of the baby, but most relationships do not survive long enough to actually marry (Furstenberg, 2014).

There are many policy issues related to growing family complexity. One issue concerning complex families is that there is still a lack of consensus about what legally constitutes family relationships. There are few family laws that apply to both married and nonmarried cohabitating or nonmarried noncohabitating families. For example, when 
married parents are seeking a divorce, states will mandate that they work out physical custody and child support arrangements. When a nonmarried cohabitating couple separates, there is not a formal legal process until one parent moves to place a child support order on the other or a parent legally demands parenting time be formally given. There are times, in some states, when a nonmarried parent applies for public assistance that may initiate a child support order on the other parent, but this process does not concern shared physical custody. One solution is to expand all marriage laws to all parenting families (Cherlin \& Seltzer, 2014); however, this solution can cause problems for those couples who do not want to be legally bound in a relationship. Another solution could be to apply laws to all parenting relationships as they apply to the care of dependents (Cherlin \& Seltzer, 2014).

Another policy consideration is how families are defined by the very programs designed to help families. For example, the Supplemental Nutrition Assistance Program (SNAP) defines a household as individuals purchasing and preparing food together for home consumption. This allows for many forms of family configurations including parents cohabitating with biological, half and even stepchildren. In contrast, programs such as Child Tax Credit (CTC) has a very legal definition of who can claim the child for tax purposes. The CTC states that the parent must provide a substantial amount of financial support. So, there could be cases where a parent does not have the child in their care for many overnights, yet still claims the child as part of their family for tax purposes based on the support the child received. There are also family definition issues with Social Security. For instance, for a spouse to receive spousal benefits, they must have been legally married for at least 10 years, nonmarried cohabiters would not be 
recognized. However, a parent's Social Security benefits can be given to a biological child, regardless if the parents are married, and sometimes even to a stepchild in certain circumstances (Meyer \& Carlson, 2014).

It is apparent from these policy examples that policies have not kept up with the changing family dynamic in the US. However, moving forward is not so easy. Some social policy experts might advocate for changing the definition of family as the family changes while other might support policy that encourages families to be less complex (promoting legal marriage, postponing nonmartial births, etc.).

\section{Coparenting in "Fragile Families"}

As stated previously, family dynamics in the U.S. has shifted to include more diverse family configurations including a growing number of parents who choose not to marry or live together. While this is true, it is also true that the income inequality in U.S. has increased over the last 20 years (Kochhar \& Cilluffo, 2017). The United States poverty line still hovers at $12 \%$ and has since the 2007 recession, but those in poverty are falling deeper into poverty with severe poverty increasing from 39\% in 1996 to $45 \%$ in 2016 (Bialik, 2017). The average income deficit is largest for a single mother as head of the family when compared to married families and families headed by a single father (Bialik, 2017). These economic issues have consequences for parenting and the coparenting relationship.

In a review of the literature, McLanahan and Beck (2010) conclude that there is a link between parents' economic resources and relationship, including the parenting relationship. They found the strongest link between a father's economic resources brought to the family and the family's behaviors including union stability, relationship 
quality, regular involvement, and engagement with children. Whereas the link with fathers was economic resources, the link with mothers was level of education. The more education the mother had, the more stable the relationship was found to be (McLanahan \& Beck, 2010).

Families with fewer economic resources are at risk for higher levels of stress, not only financial stress but also general stress that in turn is associated with higher rates of mental health issues and parenting stress (Duncan, Magnuson, \& Votruba-Drzal, 2017). These higher rates of family stressors can mean that the parents are not as available for investing, both financially and emotional, in the child's healthy development (Duncan, Magnuson, Kalil, \& Ziol-Guest, 2012). When family stress is high, it also leaves less emotional resources for the parent's couple and coparenting relationship.

\section{Coparenting in Never Married Fragile Families}

Results from the FFCWS have found that $82 \%$ of unmarried expectant fathers report being romantically involved with the mother prior to the birth of their child (McLanahan, et al., 2003). However, in the five-year post-birth data, the study found that only a third were still together at that time (McLanahan, 2009), placing them at risk for decreased chances to invest in their coparenting relationship. Children who live with single mothers and children living with mothers who have multiple partnership transitions were found to have experienced higher levels of externalizing (aggressive) and internalizing (anxiety/depressive) behaviors (Osborne \& McLanahan, 2007). They also found that children who were living in a stable cohabitating relationship did not differ from the children being raised with married parents in the same home. 
For parents who are living together, coparenting helps to reinforce the family system by strengthening the relationship each parent has with each other and the child. However, since a sizable portion of unmarried parents are also non-cohabitating, these couples may not get the same frequency of opportunities to work on the coparenting relationship. Unmarried parents who had rated their relationships as "high quality" prior to the birth of the child also reported higher levels of coparenting. Consequently, unmarried parents reported lower levels of coparenting when their partner had a child with someone else, reported high levels of depression, or had periods of incarceration. All these factors created barriers for a high-quality coparenting relationship (Carlson \& Hognas, 2011).

Recent studies have attempted to examine the impact of never married coparenting quality and outcomes in children. As a group, unmarried parents differ from the married population in several ways including: (1) unmarried parents are more likely to be younger, (2) most have a high school degree or less, (3) most identify themselves as African-American or Hispanic, (4) many report having children with more than one partner (multipartnered fertility), and (5) a high percentage of fathers in this group report a history of incarceration (Carlson, McLanahan, \& Brooks-Gunn, 2008; McLanahan et al. 2003). These findings suggest potential barriers to forming a strong coparenting relationship.

Multipartnered fertility has been found to have a significant effect on coparenting in fragile families. Carlson and Hognas (2011) noted that the level of coparenting between mothers and fathers was compromised when the parents were raising children from prior relationships. This was found to be especially significant for fathers in the 
FFCWS. The lowest levels of coparenting were reported when the father was parenting another child (other than the focal child) by another partner (other than the focal child's biological mother) (Carlson \& Hognas, 2011). Additional analysis using the FFCWS found that a mother's new relationship was associated with a decline in the biological parent's coparenting relationship between the focal child's age of 1 and 5 (Kamp Dush, Kotila \& Schoppe-Sullivan, 2011). These results bring a focus on the complexities of blended family dynamics in not just the married/divorced/re-married population but also the never-married/separated/re-partnered population.

Generally speaking, the never-married coparents can be broken into two subpopulations: (1) unmarried, cohabitating and (2) unmarried non-cohabitating. Goldberg \& Carlson $(2014,2015)$ recently conducted studies on both subpopulations. In one study, child outcomes were compared in married/cohabitating and unmarried/cohabitating coparenting relationships. A significant association was found between the coparental relationship and the child's behaviors. As couples reported higher quality in their coparenting relationship, it was found that the child had lower reported levels of externalizing and internalizing behaviors. There were no significant differences by marital status or gender of the child. The preliminary conclusion was that all children benefitted from a high-quality coparenting relationship, regardless of marital status (Goldberg \& Carlson, 2014). While these conclusions are promising in the study of coparenting relationships, there were methodological issues which call attention to gaps in the research. The first Goldberg and Carlson (2014) study, only used those couples who had been in a steady relationship for eight years. This sample may not be representative of the larger population. 
The coparenting relationship has been studied in unmarried/non-cohabitating families to reveal patterns of coparenting. Four groups emerged from their findings: (1) parents who maintained a high quality coparental relationship, (2) those who experienced a consistently low coparental relationship, (3) those who experienced improvement in their coparental relationship, and (4) those who experienced decline in their coparental relationship. Demographically speaking, they found that race/ethnicity and mother's health were found to be significant predictors of higher quality coparental relationships. Black/African American mothers who reported good health were more likely to report consistently high levels of quality coparenting. The quality of the coparents' past romantic relationship was also a strong and consistent predictor of the quality of their coparental relationship. Since a low-quality romantic relationship at the time of the child's birth was significantly associated with poor coparenting relationship, they suggest targeted coparenting interventions for this sub-population (Goldberg \& Carlson, 2015). In Goldberg and Carlson (2015) study, the researchers only use the mother's responses for coparenting measures and only used couples who had broken up their relationship in the first three years of the child's birth. This may have created a bias in that the subjects might have been the most unstable couples in the study.

\section{Coparenting and Educational Outcomes in Middle Childhood}

The quality of coparenting and its effect on child development has been reported in many research studies. However, the younger developmental stages, specifically infants to preschool age, are the subject of more research than others. In a meta-analysis by Teubert and Pinquart (2010), they found that historically most of the research examining the link between coparenting and a child's emotional and behavioral 
functioning have focused on infants, toddlers, and preschoolers. Other developmental stages of a child's development have been underrepresented in the research. Middle childhood (roughly the ages of 6-10) is one of these underrepresented areas. Furthermore, very few studies have been conducted on how the quality of the coparenting relationship specifically affects a child's school life. This present study attempts to fill this gap in the research literature.

The stage of middle childhood is an important stage to explore as the child is still very dependent on parents both emotionally and physically yet is gaining the developmental maturity to be both self-aware and situationally-aware of their family dynamics. Middle childhood is also an important time in a child's life as the child becomes increasingly aware of how they are performing in relation to their peers (Skinner \& Wellborn, 1997). Researchers have surmised that a parent's poor coparenting relationship can affect a child's sense of security which in turn can alter their healthy development (Davies \& Cummings, 1998).

Coparenting remains an important force as children enter their formal school experience. As children enter school, they are faced with new challenges such as behavioral expectations of the classroom and new peer relationships to navigate. Early research linking the coparenting relationship to academic outcomes found that supportive coparenting was positively associated with higher academic performance and higher levels of self-regulation (Brody \& Flor, 1996). If parents are not working as a coparenting team to help children navigate these early schooling years, then the child could suffer future developmental problems into adolescence (Feinberg, Kan, \& Hetherington, 2007). 
Dysfunctional coparenting relationships also puts a child at risk for unsuccessfully navigating all the tasks of a school environment that get increasingly more complicated with age. Coparent conflict was associated with an elementary child's (age 5-8) successful navigation of the school environment. The higher the observed coparenting conflict, the more at-risk the child was for internalizing symptoms and difficulties in emotionally adjusting to school situations (Sturge-Apple, Davies \& Cummings, 2010). Conversely, parents who displayed a supportive coparenting relationship were more likely to have children who displayed positive academic skills such as paying attention in class, active in class, and had good academic grades (Stright \& Neitzel, 2003). While these findings added to the coparenting literature, both the Sturge-Apple and colleagues (2010) and the Stright and Neitzel (2003) samples were compromised of families who were mostly middle-income and white.

Gender might also play a role in how coparenting affects the middle childhood developmental stage. In a study attempting to link coparenting quality with child adjustment in school-aged children, McConnell and Kerig (2002) found that coparental hostility was associated with child-reported anxiety and mother-only-reported internalizing and externalizing behaviors in sons and not daughters. Hypotheses suggested by the authors include that sons were more likely to be involved and even intervene in coparent conflict. However, like many others, this study was based on a small, convenience sample of white, middle class, married couples who had been together an average of 10 years.

Umemura and colleagues (2015) also found that gender was associated with coparenting and middle childhood school adjustment issues. In their study, they found 
that competitive coparenting observed when the child was a toddler was associated with increased ADHD symptoms in school-aged boys but not school-aged girls. For girls, there was an observed association with increase in somatization symptoms as reported by the teacher, and not parents. Once again, the sample for this study was married and mostly white and middle class.

\section{Coparenting in "Fragile Families" and Educational Outcomes}

Several researchers have started to investigate coparenting relationship quality with more diverse family constellations (e.g. Berryhill, 2017; Cabrera, Scott, Fagen, Steward-Streng \& Chein, 2012; Goldberg \& Carlson, 2015; Carlson \& Hognas, 2011). However, just a few studies have focused specifically on the child's educational outcomes and the coparenting relationship.

Recent research using the FFCWS data sets found evidence that parents with a supportive coparenting relationship had children with fewer reported behavioral problems (Goldberg \& Carlson, 2015). While one can assume these behavioral problems were also evident in an academic setting, only mother's survey responses of the child's home behaviors were used.

Berryhill (2017) is perhaps the most focused in using the FFCWS data to examine the links between coparenting and school involvement (both home based and school based). His work found that higher levels of coparenting support was associated with higher levels of mothers' and fathers' home-based school involvement and higher levels of fathers' school-based involvement. However, Berryhill's (2017) work only used parents in the sample who had maintained a relationship with their 9-year-old child. This 
may have left out a layer of parents who were not in a stable relationship, thus positively skewing the data.

Cabrera and colleagues (2012) used the Early Childhood Longitudinal StudyBirth Cohort to examine the coparenting relationship and its relationship to academic outcomes in cohabitating and married biological mothers and fathers. They found that coparenting conflict was negatively linked to a child's academic and social skills. While Cabrera and colleagues did add to the research connecting the coparenting relationship to academic outcomes, it only gathered data at the preschool level.

\section{Literature Review Summary}

The review of the literature related to the coparenting relationship and educational outcomes revealed several gaps in the research. Firstly, previous research investigating family dynamics and a child's school success has focused on the married, then divorced population or on the single mother and child dyad (Carlson \& McLanahan, 2002). Consequently, samples have largely been white, middle class, and female respondents (McLanahan, 2009). Additionally, these sampling techniques leave out a significant amount of the coparenting population who is cohabitating nonmarried or noncohabitating nonmarried yet still involved in the child's life. This current study attempts to address these gaps by using a sample that is more diverse racially/ethnically, socioeconomically, and by coparenting relationship/union status.

Secondly, the proposed study will also contribute to the literature through its use of educational outcomes as a primary outcome. This represents a departure from previous research in which educational outcome markers (e.g. grades) have been used as a covariable or control variable. Previous work has also relied on the mothers' self-report 
measures of family function. This means that the mother could be critical of the father in the dyadic relationship, but the father was not asked for input. The FFCWS is useful in addressing this gap in the literature because it features the largest nationally representative sample of unwed fathers responding to survey collection (Reichman, Teitler, Garfinkel, \& McLanahan, 2001).

Thirdly, this current study will add to the research by focusing on the middle childhood developmental stage. Much of the prior research had focused on coparenting dynamics during the birth to preschool developmental stages. The current study will use data from 9-year-olds, their biological mothers and fathers, and the focal child's teacher. Lastly, this current study will attempt to address methodological issues surrounding the construct of coparenting. In studies examining the coparenting construct and using the FFCWS data sets, other researchers (e.g. Berryhill, 2017; Goldberg and Carlson, 2015; Carlson, McLanahan, \& Brooks-Gunn, 2008) used a small series of questions (3-6 items) taken from the survey related to coparenting behaviors but did not tie them back to any one theoretical model of coparenting. 


\section{CHAPTER 3: METHODS}

This chapter begins with a review of the purpose of the study and the research questions. It is then followed by an overview of the Fragile Families and Child Wellbeing Study (FFCWS), followed by the population and sample used in this study. Next, procedures and measures are detailed as well as an explanation of independent and dependent variables that are under examination. Finally, analytical methods are discussed.

\section{Purpose of the Study}

The aim of this research is to examine father's perceptions of the coparenting relationship in diverse family systems using Feinberg's Model of Coparenting Components (Feinberg, 2003) and their association with children's educational outcomes.

\section{Research Questions}

Research Questions include:

1a. Are there differences in father's perceptions of coparenting by relationship status (with child's mother)?

1b. Are there significant differences when controlling for background factors (education, poverty level, age, race/ethnicity)?

2a. Are there differences in father's perceptions of coparenting by time spent with the child?

$2 \mathrm{~b}$. Are there significant differences when controlling for background factors (education, poverty level, age, race/ethnicity)?

3a. Are father's perceptions of coparenting associated with the focal child's school success (academic and behavioral measures) at age 9?

$3 \mathrm{~b}$. Are there significant differences when controlling for background factors (education, poverty level, age, race/ethnicity)? 


\section{Figure 2: Diagram of Full Model}

Father's Independent

\& Control Variables

Family Process Variables

Child School

Outcomes

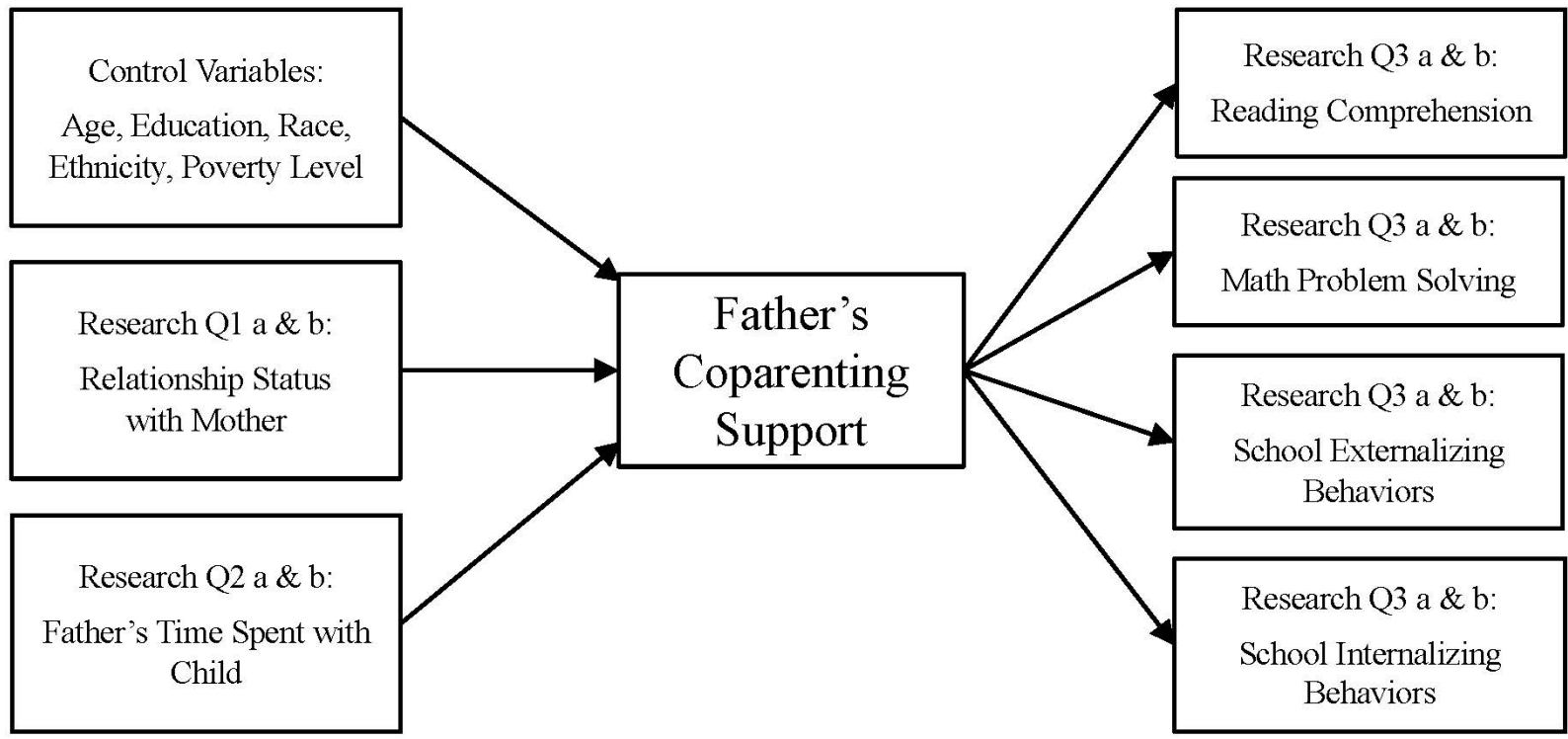

\section{Data Source: Fragile Families and Child Wellbeing Study (FFCWS)}

The Fragile Family and Child Wellbeing Study (FFCWS) is a national, longitudinal study following a cohort of 4,898 births between 1998 and 2000 (McLanahan, 2009). Sara McLanahan of Princeton University and Irwin Garfinkel of Columbia University are the principal investigators for the study. They set out to address a research gap that had been noted as a limitation by many family researchers. Most of the research on families to date had been conducted on white, married, middle class families. Given the shift in family structure discussed earlier, it was important to collect data from a more diverse population that represented the changing family more specifically to include minority families, low income families and those choosing not to marry. 
The study was designed to explore the following research questions: "What are the capabilities of unmarried parents when their child is born, especially fathers? What is the nature of relationships in fragile families at birth? How do relationships change over time? How do parents and children fare in fragile families” (McLanahan, 2009 p.3). Since the start of the FFCWS, there have been over 600 studies published using this data set to examine the various aspects of the fragile family ("Key Findings for Policy Makers", 2016).

The FFCSW study is funded through a variety of government/public agencies (e.g. National Science Foundation and the U.S. Department of Health and Human Services) as well as private foundations (e.g. Robert Wood Johnson Foundation and the Foundation for Child Development). Additionally, research funding was provided by the Eunice Kennedy Shriver National Institute of Child Health and Human Development to collect data at the child's age of 15 (Ret from: http://fragilefamilies.princeton.edu/about). A complete list of funding sources for the study can be found at:

https://fragilefamilies.princeton.edu/about/funders.

\section{FFCWS Population and Sample}

Since the FFCWS set out to explore the changing family structure, the study oversampled nonmarital births in a 3:1 ratio $(N=4,898$, with 3,710 unmarried parents and 1,188 married parents). Initial interviews were conducted with both mothers and fathers (when present) in the hospital following the birth of the focal child (Reichman et al., 2001). The core study has collected follow up data at the child's age 1 , age 3 , age 5 , age 9, and age 15. Data gathered from baseline to year 9 are available (with permission) 
through the Office of Population Research at Princeton university. The analyses for this proposed study will be conducted using the 9-year-old data from the FFCWS.

The sampling strategy included recruiting couples from 20 cities and 75 hospitals across the United States. Initial interviews were conducted in the hospitals so as to take advantage of the "magic moment" right after the child's birth (McLanahan et al., 2003). Cities in the sampling included: Oakland, CA; San Jose, CA; Jacksonville, FL; Chicago, IL; Indianapolis, IN; Baltimore, MD; Boston, MA; Detroit, MI; Newark, NJ; New York, NY; Toledo, OH; Philadelphia, PA; Pittsburgh, PA; Nashville, TN; Austin, TX; Corpus Christi, TX; San Antonio, TX; Norfolk, VA; Richmond, VA; and Milwaukee, WI. The cities were originally selected based on the social policies and labor conditions within those cities (Reichman et al., 2001). All cities included had populations over 200,000. The FFCWS used a random stratified sampling to select the initial participants. The sampling occurred in three stages: (1) sampling within the cities, (2) sampling within the hospitals, and (3) sampling the births within each hospital.

The original baseline survey included 4898 mothers and 3830 fathers. In year 1, 4364 mothers completed the survey and 3379 fathers. Cases were considered ineligible for continuation in the study if a parent was deceased, the child was deceased, the child was adopted, or if DNA results proved that the original father identified was not the biological father. In year 3, 4231 mothers responded, and 3299 fathers responded to the survey. In year 3, a primary care giver survey was also added and 3288 responded. In year 5, 4139 mothers responded, 3159 fathers responded, and 2989 primary caregivers responded. In Year 5, kindergarten teachers were added, and 1039 of the kindergarten teachers completed their surveys. In year 9, 3515 biological mothers, 2652 biological 
fathers, 3630 primary caregivers, and 2254 teachers completed the survey instruments. Focal child interviews were added at age 9, and 3377 children completed this interview. Year 9 was used in this particular study in order to represent the middle child developmental stage. The year 5 (one data set previous) data did not include academic measures. Year 15 (one data set later) did not include questions on coparenting support. Surveys used in the analysis for this study included biological father surveys, in-home child assessments (reading comprehension and applied math assessments), and teacher surveys (school internalizing and externalizing behaviors).

The sample for the year 9 survey included biological mothers $(N=3515)$, biological fathers $(N=2652)$, and teachers $(N=2254)$. Since this current study is focused on biological fathers who are coparenting, the original proposed analytic sample was 2652 biological fathers. As noted in the FFCWS data guide for year 9, there were children who did not complete the full battery of academic assessments at the in-home interview (Bendheim-Thoman Center for Research on Child Wellbeing, 2005). Missing data analysis revealed that only $41 \%$ of the cases with biological father coparenting survey items also had completed the in-home child academic assessments. Additionally, 27 cases were removed where it was indicated that the biological father was unknown or had a romantic non-cohabitating relationship with the mother but did not spend time with the child. After data cleaning, a total of 1255 cases were used as the final analytic sample.

\section{Rationale for Using the FFCWS in the Current Study}

The FFCWS was specifically selected for this study to address the following reasons: (1) It has a large sample size which allows for the statistical power necessary for 
analysis. This will also allow for certain variables to be controlled and therefore be able to isolate variables of interest (e.g. coparenting relationship, union status). (2) FFCWS is one of the largest samples of diverse kinds of family constellations. As a social worker, advocating for diverse populations is core to the profession's values. The FFCWS allows for the voices and experiences of underrepresented populations to be considered in contemporary family research. More specifically, this sample is diverse ethnically, socioeconomically, and family union statuses. (3) Lastly, the FFCWS has captured the voice of low-income fathers, a voice often missing from coparenting studies. In previous FFCWS research (e.g. Marchand-Reilly \& Yaure, 2019; Karberg \& Cabrera, 2017; Goldberg \& Carlson, 2015; Kamp Dush et al., 2011), only the mother's voice was used to speak for the coparenting relationship of the family even though the same coparenting survey items were administered to fathers in the study. This not only seems unfair to fathers, but also potentially skewing the foundational data that others have built subsequent research upon.

\section{Research Design}

This study is a quantitative research design using secondary data from the Fragile Families and Child Wellbeing Study (FFCWS). The FFCWS is a nonexperimental, longitudinal design that follows a cohort of children and their families from birth to age 15. Kerlinger and Lee (2000) describe nonexperimental research as an inquiry in which the researcher does not have direct control of the independent variables because they are not inherently manipulable. For the purpose of this particular study, the design will be cross-sectional examining the cohort in year 9 .

\section{Procedures}

\section{Mother and Father Interviews}


The mother was interviewed in person in the hospital within 48 hours of the birth of the focal child. This interview was the baseline and then subsequent phone interviews with the mother occurred when the focal child was 1,3,5 and 9 years old. Fathers were initially interviewed either in the hospital, if available, or by phone within a week of the birth of the focal child, then had follow up interviews by phone the same as mother when the focal child was 1,3,5 and 9. Phone interviews lasted an average of 42 minutes. If mothers or fathers were not able to be reached by phone, a trained field researcher would attempt an interview in person. Beginning with year 3 data, mothers and children were interviewed and assessed in the home setting. In Year 9, biological mothers were paid $\$ 30$ and biological fathers were paid $\$ 75$ for completing in person or telephone interviews (Bendheim-Thoman Center for Research on Child Wellbeing, 2005).

\section{In-Home Focal Child Assessment}

The in-home focal child assessment included the mother's interview, an assessment by the trained researchers, and the child's self-report (starting at age 9). These assessments were conducted in the home and lasted an average of 60 minutes (Bendheim-Thoman Center for Research on Child Wellbeing, 2014b). The mother's report on the child's behavior was collected at 1 year for temperament, and 3,5,9 years for other areas like nutritional and health, behaviors, emotional development, discipline, and family routines. The focal child's report of their own behavior was collected at year 9 and included information on their parents, siblings, routines, school, discipline, and their own behaviors. Additionally, child assessments for the Woodcock Johnson Passage Comprehension (WJ Subtest 9) and Applied Problems (WJ Subtest 10) were collected during the home visit in year 9. Families were paid $\$ 65$ for completing the in-home 
interview and child assessment (Bendheim-Thoman Center for Research on Child Wellbeing, 2005).

\section{Teacher Interviews}

Classroom teachers were given surveys to mail back at years 5 and 9 . These surveys included items such as the child's academic skills, classroom behavior including school internalizing and externalizing behaviors, school environmental questions, and their own background as teachers.

\section{Variables \& Measures}

\section{Coparenting Support Variables}

In previous waves (child age 1,3,5), mothers were asked a series of six questions that focused on the coparenting relationship. These six items have been used by various researchers (e.g. Choi \& Belcher, 2019; Karberg \& Cabrera, 2017; Goldberg \& Carlson, 2015) to isolate the executive sub-system of the coparenting relationship. These questions are as follows:

1. When father is with child, he acts like the father you want for your child.

2. You can trust father to take good care of child.

3. He (bio-father) respects the schedules and rules you make for the child.

4. He (bio-father) supports you in the way you want to raise child.

5. You and father talk about problems that come up with raising child.

6. You can count on father for help when you need someone to look after child for a few hours.

However, the purpose of this study was to examine fathers' perceptions of coparenting. In Wave 5 (child age 9), only three items from the previous six coparenting items asked 
of mothers were asked of fathers. The three items used for the fathers' perception of coparenting measures in this study were:

1. You (father) can trust mother to take good care of child.

2. She (mother) supports you in the way you want to raise child.

3. You and mother talk about problems that come up with raising child.

All questions used the following as possible responses: $1=$ always true, $2=$ sometimes true, 3 = rarely true or $4=$ never true. The responses were reverse coded for analysis so that higher numerical scores indicated higher coparenting support ratings. A Cronbach Alpha analysis was conducted to assess internal reliability for the three father coparenting survey items. Scale reliability was .804 which is considered in the acceptable range for social sciences (Cronbach, 1951; "What Does Cronbach's Alpha Mean”, n.d.). For the purpose of this study, the three father coparenting questions were combined into a scale of all the averaged responses. After examining the frequency of the responses, father's coparenting ratings were then grouped into three categories which included (1) low level of perceived coparenting support from mother, (2) medium level of perceived coparenting support from mother, and (3) high level of perceived coparenting support from mother.

\section{Educational Outcome Variables \\ Child Educational Levels}

The focal child, at age 9, was tested on several educational assessments including reading comprehension and applied math skills. Reading comprehension and applied math skills were assessed using the Woodcock Johnson Passage Comprehension and Applied Problems (Woodcock, McGrew \& Mather, 2001). The Passage Comprehension 
includes reading a short passage and identifying a missing word based on the context of the passage with scores ranging from 1 to 135 . The items become more difficult as the assessment progresses with longer passages, more difficult levels of vocabulary, and more complex syntax and semantics cues. The Applied Problems subtest assesses the focal child's ability to analyze and solve math problems. The assessment items include both problem solving and calculation with scores ranging from 1 to 143. Items on the Applied Problems subtest also increase in difficulty. Standardized scores on both the reading comprehension and applied math skills ranged from 1 to 159. (BendheimThoman Center for Research of Child Wellbeing, 2013).

\section{Child Internalizing \& Externalizing School Behaviors}

Data were collected about the child's internalizing and externalizing school behaviors using self-report survey items rated by the child's teacher using the Social Skills Rating System (SSRS: Greshman \& Elliott, 1990). Items used from the SSRS used a 4-point Likert scale in which $0=$ never, $1=$ sometimes, $2=$ often, and $3=$ very often. This part of the survey consisted of six items for internalizing behaviors and six items for externalizing behaviors. Internalizing behaviors measured included: having low selfesteem, appearing lonely, showing anxiety with a group of children, being easily embarrassed, liking to be alone, and acting sad/depressed. Externalizing behaviors measured included: fighting with others, threatening/bulling others, arguing with others, talking back to adults when corrected, getting angry easily, and having temper tantrums. Reliability analysis were conducted for both the six-item internalizing scale and the sixitem externalizing scale. Cronbach's Alpha revealed an internal reliability score of .920 for the items in the externalizing scale and a score of .858 for the items in the 
internalizing scale. Both of these scores are considered acceptable for further analysis (Cronbach, 1951; “What Does Cronbach’s Alpha Mean", n.d.). After examining the frequency of the survey responses, it was noted that the teacher responses were skewed towards positive responses. The decision was made to create a dichotomized variable for externalizing and internalizing child behaviors. The dichotomized variable was created using a cut off score at the approximate median, based on response frequency analysis. The externalizing and internalizing survey results were divided into two categories for analysis which included (1) high internalizing (range of 1.5 to 3)/externalizing behaviors (range of 1.4 to 3 ) and (2) low internalizing (range of 0 to 1.499)/externalizing (range of 1.5 to 3 ) behaviors.

\section{Control Variables}

In order to isolate the effect of coparenting perceptions on school success, various control or background variables were used as having been identified in previous literature (e.g. Choi \& Becher, 2019; Fagan \& Cherson, 2017). These control variables include fathers' age (measured continuously), race/ethnicity (measured categorically: NonHispanic White, Non-Hispanic Black, Hispanic, Other), level of education (measured categorically: Less than high school, High school or equivalent, Some college/technical school, College or graduate school), and poverty level (measured continuously as an income to poverty ratio, the ratio of total household income to the official poverty thresholds as determined by the U.S. Census Bureau, Bendheim-Thoman Center for Research on Child Wellbeing, 2014b). 


\section{Analytic Strategy}

First, descriptive statistics were run for the independent, dependent, and control variables for each of the research questions. Bivariate correlations were completed to examine the following relationships: (1) relationship status by fathers coparenting and (2) time spent with child and fathers coparenting. Next, pairwise correlations were completed for each of the school success measures and father's coparenting. Lastly, regression tables were completed for each of the dependent variables used in the research questions.

\section{Research Question 1}

Research question 1 examines the differences in father's perception of coparenting by relationship status and then examines statistically significant differences while controlling for background factors.

IV: The independent variable is relationship status measured as a categorical variable which includes (1) married, (2) romantic cohabitating, (3) friends (non-cohabitating), (4) separated/divorced/widowed, or (5) no relationship. Background control variables include: Father's race/ethnicity (Non-Hispanic White, Non-Hispanic Black, Hispanic; Other), Father's education level (Less than high school, High school or equivalent, Some college or trade school, College or graduate school), Father's age, and Father's income to poverty ratio.

DV: The dependent variable is father's coparenting measured as a continuous variable as a mean of three coparenting questions which were measured on a scale from 1-4 and reverse coded to that lower responses corresponded to lower coparenting reports. This score was then divided into three categories of (1) low, (2) medium, and (3) high levels of perceived coparenting support. 
H1: Fathers who are married or cohabitating with their child's mother will report higher coparenting support levels.

The hypothesis for research question 1 was tested using an ANOVA comparing fathers' coparenting scores by relationship status groups to determine if there were significant differences found between the different relationship groups. Then, I added control variables and ran a multinomial logistic regression using coparenting support categories (low, medium, high) to test whether these differences were still significant once the control variables are added.

\section{Research Question 2}

This question examines fathers' perception of coparenting by time spent with the child and then examines statistically significant differences while controlling for background factors.

IV: The independent variable is time spent with child measured as a categorical variable which includes: (1) all/most of the time, (2) half/some of the time/weekends, or (3) none of the time. Background control variables include: Fathers' race/ethnicity (Non-Hispanic White, Non-Hispanic Black, Hispanic, Other); Fathers' education level (Less than high school, High school or equivalent, Some college or trade school, College or graduate school), Fathers' age, and Fathers' income to poverty ratio.

DV: The dependent variable is fathers' coparenting measured as a continuous variable as a mean of three coparenting questions which were measured on a scale from 1-4 and reverse coded to that lower responses corresponded to lower coparenting reports. This score was then divided into three categories of (1) low, (2) medium, and (3) high levels of perceived coparenting support. 
H2: Fathers who spend more time with their child will report higher coparenting support levels.

This hypothesis was tested by performing an ANOVA comparing fathers' coparenting scores by time spent with child groupings to determine if there were significant differences found between the different time spent groups. Next, control variables were added and a multinomial logistic regression was conducted using coparenting support categories (low, medium, high) to test whether these differences were still significant once the control variables are added.

Research Question 3

The final research question examines the relationship between fathers' perception of coparenting and the child's school outcomes.

IV: The independent variable is fathers' coparenting measured as a continuous variable as a mean of three coparenting questions which were measured on a scale from 1-4 and reverse coded to that lower responses corresponded to lower coparenting reports. This score was then divided into three categories of (1) low, (2) medium, and (3) high levels of perceived coparenting support. Background control variables include: Fathers' race/ethnicity (Non-Hispanic White, Non-Hispanic Black, Hispanic, Other); Fathers’ education level (Less than high school, High school or equivalent, Some college or trade school, College or graduate school), Fathers' age, and Fathers' income to poverty ratio. DV: The dependent variables were the four school outcomes measures. Two outcomes were continuously measured variables. These include reading comprehension and math problem solving. Two additional measures provide social skill levels of the children as reported by the classroom teacher. There were two scales including externalizing social 
skills and internalizing social skills. These scales were divided into two categories for analysis which included (1) high internalizing/externalizing behaviors and (2) low internalizing/externalizing behaviors.

H3: Fathers reporting a higher level of coparenting relationship with the child's mother will have children who were finding higher success in school as measured by standardized academic tests (higher reading and math scores) and behavior measures (lower rates of displayed externalizing and internalizing behaviors at school).

Ordinal least squares (OLS) regression was conducted as the analysis for research question 3 for the reading comprehension and math problem solving parts of the model as they are continuous variables. For the dependent variables that were binary (externalizing and internalizing social skills), binary logistic regression analyses were conducted. Finally, the control variables were added to test whether these differences were still significant once the control variables were added. 


\section{CHAPTER 4: RESULTS}

This chapter first presents descriptive statistics of social and demographic characteristics of the final analytical sample. Next, it presents the bivariate and multivariate results for research questions 1,2 and 3.

\section{Preliminary Descriptive Statistics}

Table 1.1 presents descriptive statistics of the analytic sample. The final analytic sample for this study was 1255 cases. The average age of fathers was $37.39(s d=7.22)$ with a range of 26-76. Five hundred eighty-eight ( $n=588 ; 46.85 \%)$ fathers included in this study identified as Non-Hispanic Black, followed by 337 fathers who identify as NonHispanic White (26.85\%), 284 who identified as Hispanic (22.63\%), and 46 who identified as "other" race/ethnicity (3.67\%).

Another demographic variable used as a control in the analysis was father's level of education. Past researchers (e.g. Choi \& Becher, 2019; Fagan \& Cherson, 2017), have included the parent's level of education when examining the child's education attainment and coparenting and found that fathers with less education report lower coparenting. In terms of education level for this study, 272 (21.67\%) of fathers had less than a high school education. Three hundred twenty-eight $(n=328)$ fathers with a high school or equivalent degree made up $26.14 \%$ of the sample. Four hundred fifty-six $(n=456)$ fathers with some college or technical school made up the largest percentage with $36.33 \%$ reporting this level of education. Lastly, 199 (15.86\%) of fathers in this study report having a college or graduate school degree. 
Additionally, the fathers' income to poverty ratio was used as a control variable in the analysis. In the FFCWS study, the constructed variable for this measure is the ratio of the total household income to official poverty thresholds as established by the U.S. Census Bureau (Bendheim-Thoman Center for Research on Child Wellbeing, 2014b). For this study, fathers' income to poverty ratio ranged from $0-28.85$ with an average of 2.79 and standard deviation of 2.62 units on this ratio scale. Any father with an income to poverty ratio of 0 was determined to be at or below the poverty level for that household. Numbers higher than 0 referred to the surplus of income in the father's household over the official poverty threshold (Ret: https://www.census.gov/topics/incomepoverty/poverty/guidance/poverty-measures.html). 
Table 1.1 Descriptive Statistics for Study Variables $(\mathrm{N}=1255)$

\begin{tabular}{|c|c|c|c|}
\hline \multicolumn{4}{|l|}{ Demographic Control Variables } \\
\hline \multicolumn{4}{|l|}{ Father Education Level } \\
\hline College/Graduate School & $\mathrm{n}=199$ & $\%=15.86$ & \\
\hline Some College/Technical School & $\mathrm{n}=456$ & $\%=36.33$ & \\
\hline High School or equivalent & $\mathrm{n}=328$ & $\%=26.14$ & \\
\hline Less than High School & $\mathrm{n}=272$ & $\%=21.67$ & \\
\hline \multicolumn{4}{|l|}{ Father Ethnicity/Race } \\
\hline Other & $\mathrm{n}=46$ & $\%=3.67$ & \\
\hline Hispanic & $\mathrm{n}=284$ & $\%=22.63$ & \\
\hline Non-Hispanic Black & $\mathrm{n}=588$ & $\%=46.85$ & \\
\hline Non-Hispanic White & $\mathrm{n}=337$ & $\%=26.85$ & \\
\hline \multicolumn{4}{|l|}{ Relationship Status } \\
\hline Married & $\mathrm{n}=544$ & $\%=43.35$ & \\
\hline Romantic Cohabitating & $\mathrm{n}=166$ & $\%=13.23$ & \\
\hline Friends & $\mathrm{n}=240$ & $\%=19.12$ & \\
\hline Separated/Divorced/Widowed & $\mathrm{n}=141$ & $\%=11.24$ & \\
\hline No Relationship & $n=164$ & $\%=13.07$ & \\
\hline \multicolumn{4}{|l|}{ Time Spent with Child } \\
\hline All or Most of the time & $\mathrm{n}=771$ & $\%=61.43$ & \\
\hline Half/some of the time/weekends & $\mathrm{n}=298$ & $\%=23.75$ & \\
\hline None of the time & $\mathrm{n}=186$ & $\%=14.82$ & \\
\hline Father's Age & Range $=25-76$ & Mean $=37.39044$ & $\mathrm{SD}=7.222853$ \\
\hline Father's Income to Poverty Ratio & Range $=0-28.85408$ & Mean $=2.97738$ & $\mathrm{SD}=2.621042$ \\
\hline Father Coparenting Questions & Range & Mean & SD \\
\hline $\mathrm{Q} 1=$ Trusts Mother & $1-4$ & 3.806375 & .5533348 \\
\hline Q2=Mother Supports Me & $1-4$ & 3.49004 & .8182651 \\
\hline Q3=Talks with Mother about Child & $1-4$ & 3.542629 & .8288408 \\
\hline Coparenting Scale (Mean Q1,Q2, Q3) & $1-4$ & 3.613015 & .6311293 \\
\hline
\end{tabular}


Father Coparenting Categories

Low Coparenting Support

Medium Coparenting Support

High Coparenting Support

\section{Child Outcome Variables}

Child Reading Score

Child Math Score

Child Externalizing Behaviors

(School)

Child Internalizing Behaviors

(School)

Child Externalizing Scale Results

Externalizing High

Externalizing Low

$$
\begin{array}{ll}
\mathrm{n}=192 & \%=15.30 \\
\mathrm{n}=378 & \%=30.12 \\
\mathrm{n}=685 & \%=54.58
\end{array}
$$
$\%=15.30$
$\%=30.12$
$\%=54.58$

Range

$1-135$

$1-143$

$1-4$

$1-3.8333$

Mean

\section{SD}

94.83665

99.97211

1.4949

13.9833

16.9072

.6313721

1.573679

.5296293

$\begin{array}{ll}\mathrm{n}=506 & \%=40.32 \\ \mathrm{n}=749 & \%=59.68\end{array}$

$n=639$

$\%=50.92$

$\%=49.08$

$\mathrm{n}=616$

$\%=49.08$

Internalizing High 


\section{Bivariate Correlations}

Table 1.2 shows bivariate correlations among all the main study variables used in the three research questions. The fathers' average coparenting score was significantly negatively correlated with every category with the exception of child's reading $(r(1253)$ $=.065, p<.05)$ and math scores $(r(1253)=.089, p<.01)$ in which it was significantly positively correlated. When the coparenting score was broken into categories, it was also significantly negatively correlated with every category except the child's reading $(r$ $(1253)=.085, p<.01)$ and math $(r(1253)=.090, p<.01)$ scores which were found to be significantly positively correlated to coparenting categories. The time the father spent with his child was significantly positively correlated to the relationship status with the child's mother $(r(1253)=.704, p<.01)$ and the child's externalizing behaviors at school $(r(1253)=.175, p<.01)$. The time the father spent with the child was significantly negatively correlated with the child's reading $(r(1253)=-.143, p<.01)$ and math scores $(r(1253)=-.128, p<.01)$. The relationship status between the father and the mother was also significantly negatively correlated with the child's reading $(r(1253)=-.158, p<.01)$ and math scores $(r(1253)=-.159, p<.01)$; however, the relationship status was found to be significantly positively correlated to the externalizing $(r(1253)=.216, p<.01)$ and internalizing $(r(1253)=.080, p<.01)$ behaviors the child demonstrated at school. The child's reading score was positively significantly correlated to the child's math score but was significantly negatively correlated to time spent with father, the father-mothers' relationship status, and the internalizing and externalizing school behaviors. Likewise, the child's math score was significantly positively correlated to the child's reading score but was significantly negatively correlated to time spent with father, the father-mothers' 
relationship status, and the child's internalizing and externalizing school behaviors. The child's externalizing behaviors at school were significantly negatively correlated with fathers' coparenting support as well as the child's reading and math scores. However, the child's externalizing behaviors were found to be significantly positively correlated with the time spent with the child's father, the relationship status between the father and mother, and internalizing behaviors at school. Similarly, the child's internalizing behaviors at school were significantly negatively correlated with fathers' coparenting support as well as the child's reading and math scores. The internalizing behaviors were significantly positively correlated with the time spent with the child's father, the relationship status between the father and mother, and internalizing behaviors at school. 
Table 1.2 Bivariate Correlations

\begin{tabular}{|c|c|c|c|c|c|c|c|c|c|c|}
\hline & 1 & 2 & 3 & 4 & 5 & 6 & 7 & 8 & 9 & 10 \\
\hline $\begin{array}{l}\text { 1. F Coparenting } \\
\text { Mean }\end{array}$ & 1.000 & & & & & & & & & \\
\hline $\begin{array}{l}\text { 2. F Coparenting } \\
\text { Categories }\end{array}$ & $.866^{* *}$ & 1.000 & & & & & & & & \\
\hline 3. Time w/Child & $-0.354 * *$ & $-0.388 * *$ & 1.000 & & & & & & & \\
\hline 4. Rela w/mother & $-0.478 * *$ & $-0.486 * *$ & $0.704 * *$ & 1.000 & & & & & & \\
\hline 5. Child's Reading & $0.065^{*}$ & $0.085^{* *}$ & $-0.143 * *$ & $-0.158 * *$ & 1.000 & & & & & \\
\hline 6. Child's Math & $0.089 * *$ & $0.090 * *$ & $-0.128 * *$ & $-0.159 * *$ & $0.636^{* *}$ & 1.000 & & & & \\
\hline 7. Child's Ext Scale & $-0.122 * *$ & $-0.135 * *$ & $0.175 * *$ & $0.191 * *$ & $-0.226^{* *}$ & $-0.174 * *$ & 1.000 & & & \\
\hline 8. Ext Scale Binary & $-0.156^{* *}$ & $-0.160 * *$ & $0.189 * *$ & $0.216^{* *}$ & $-0.222 * *$ & $-0.184 * *$ & $0.767 * *$ & 1.000 & & \\
\hline 9. Child's Int Scale & -0.017 & -0.007 & 0.052 & $0.080 * *$ & $-0.218 * *$ & $-0.236 * *$ & $0.353 * *$ & $0.313^{*}$ & 1.000 & \\
\hline 10. Int Scale Binary & -0.052 & -0.045 & 0.045 & $0.080 * *$ & $-0.186^{* *}$ & $-0.194 * *$ & $0.310 * *$ & $0.284^{*}$ & $0.758^{*}$ & 1.000 \\
\hline
\end{tabular}




\section{Results for Research Q1:}

A one-way ANOVA was conducted to determine if fathers' perception of coparenting was different for fathers in various relationship categories with their child's mother. The relationship categories consisted of (1) married, (2) romantic cohabitating, (3) friends, (4) separated/divorced/widowed, and (5) no relationship. There was a statistically significant difference between groups as determined by one-way ANOVA ( $F$ $(4,1250)=95.70, p=.000)$. A Tukey post-hoc test revealed that fathers' coparenting perceptions were statistically significant different for all groups with the exception of Married (group 1) when compared to Romantic/Cohabitating (group 2). Results were also not significant for Friends (group 3) when compared to Separated/Divorced/Widowed (group 4). The fathers who rated their coparenting as highest were in the married category with romantic cohabitating having the next highest coparenting rating. In sum, fathers who co-reside while coparenting their child rate their coparenting support significantly higher than all other groups not having physical access to their child.

A multinomial logistic regression was used to predict the coparenting level of fathers in the study. Fathers' coparenting support (mean scale) was divided into three categories (low, medium, high) with low coparenting support being the reference category. The independent variable was relationship category with married as the reference category. Four other independent variables (education, race/ethnicity, age, and poverty level) were added as control variables with relationship status categories. Results of the multinomial logistic analysis indicated that the five-predictor model provides a 
statistically significant prediction of coparenting support level, -2 Log Likelihood = $1042.233, X^{2}(24, N=1255)=373.15, p<.001$.

Medium Coparenting Versus Low Coparenting by Relationship Status: As shown in Table 1.4 below, results reveal that cohabitating fathers had a $70 \%$ lower relative risk of reporting medium coparenting versus low coparenting support when compared to married fathers. Fathers in the "friend" category and the "separated, divorced, widowed" category had a 94\% lower relative risk of reporting medium coparenting versus low coparenting support when compared to the married group. Fathers who described themselves as having "no relationship" with their child's mother had a 97\% lower relative risk of reporting medium coparenting versus low coparenting support when compared to married fathers.

The majority of the control variables were found to not be statistically significant in the medium versus low coparenting support categories. The only exceptions were minority fathers in the Non-Hispanic Black category. Non-Hispanic Black fathers had two (2) times greater relative risk to report medium coparenting support versus low coparenting support when compared to Non-Hispanic White fathers.

\section{High Coparenting Versus Low Coparenting by Relationship Status: Cohabitating} fathers had an $84 \%$ lower relative risk of reporting high coparenting versus low coparenting support when compared to married fathers. Fathers in the "friend" category reported a $98 \%$ lower relative risk of reporting high coparenting versus low coparenting support when compared to married fathers. Fathers in the "separated, divorced, widowed" and "no relationship" categories had a 99\% lower relative risk of reporting high coparenting versus low coparenting support when compared to the married group. 
Once again, the majority of the control variables were found to not be statistically significant in the high versus low coparenting support categories. The two exceptions were minority fathers in the Non-Hispanic Black and Hispanic categories. Non-Hispanic Black fathers were 2.23 times greater relative risk to report high coparenting support versus low coparenting support when compared to Non-Hispanic White fathers. Hispanic fathers were 2.29 times greater relative risk to report high coparenting support versus low coparenting support.

Since minority fathers were showing statistical significance in the overall model, additional interaction analysis between relationship status and father's race/ethnicity revealed no statistically significant levels. The results of research question 1 supports the original hypothesis that fathers in married or cohabitating relationships with the child's mother were significantly more likely to report higher coparenting levels.

Chart 1.1, Table 1.3, and Table 1.4 display visual representations of results for research question 1. Chart 1.1 presents the predicted probabilities of coparenting by relationship status. Table 1.3 presents the results from the statistical analysis from the ANOVA and Table 1.4 presents the results from the multinomial logistic regression. 


\section{Chart 1.1 Predicted Probability of Coparenting by Relationship Status}

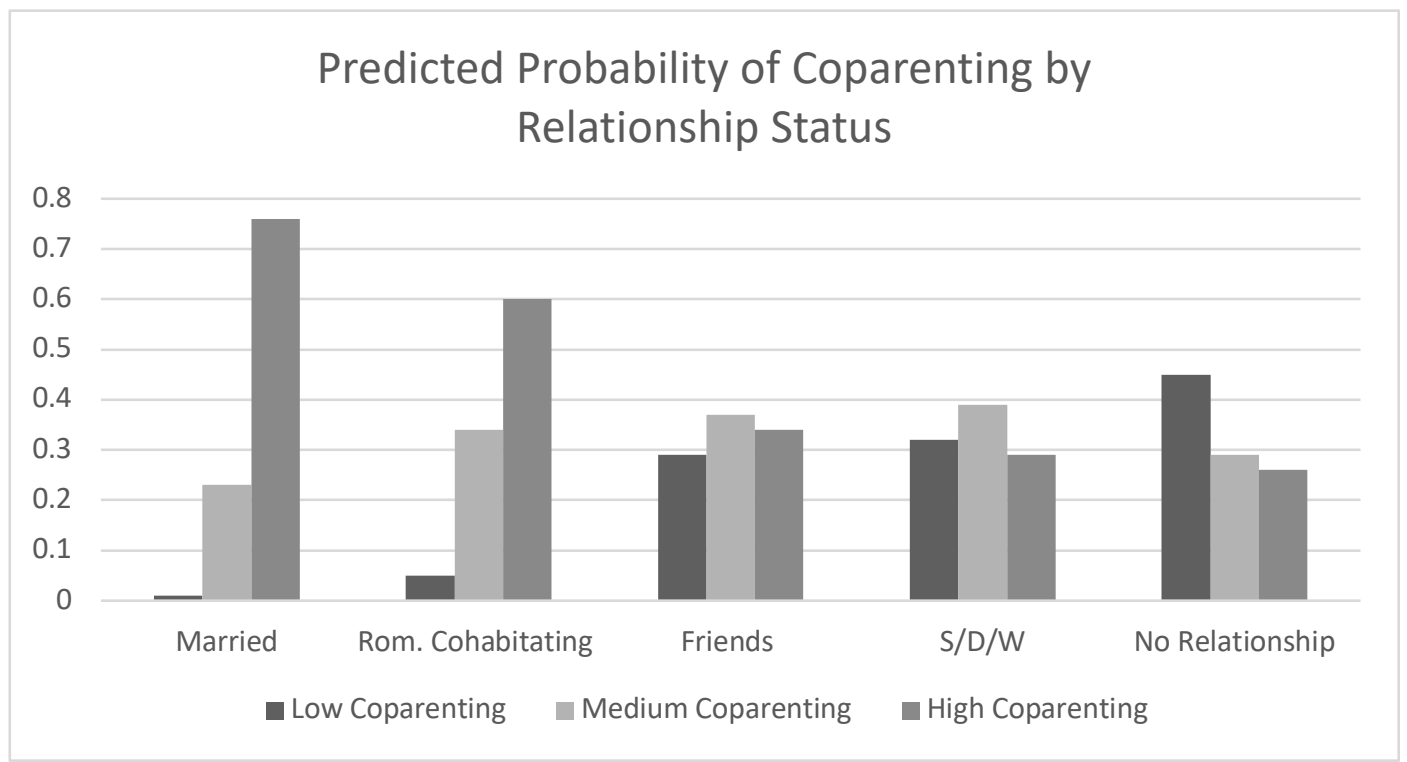

Table 1.3: Research Question 1 ANOVA with Tukey post hoc results

\begin{tabular}{|c|c|c|c|c|c|c|c|}
\hline & $\mathrm{n}$ & Mean & $\mathrm{SD}$ & Tukey's & omparisons & & \\
\hline & & & & 1 & 2 & 3 & 4 \\
\hline $1=$ Married & 544 & 3.88 & .247 & & & & \\
\hline $\begin{array}{l}2=\text { Romantic/ } \\
\text { Cohabitating }\end{array}$ & 166 & 3.78 & .383 & .184 & & & \\
\hline $3=$ Friends & 240 & 3.42 & .706 & $.001 * *$ & $.001 * *$ & & \\
\hline $\begin{array}{l}4= \\
\text { Sep/Div/Wid }\end{array}$ & 141 & 3.34 & .703 & $.001 * *$ & $.001 * *$ & .681 & \\
\hline $\begin{array}{l}5=\text { No } \\
\text { Relationship }\end{array}$ & 164 & 3.05 & .916 & $.001 * *$ & $.001 * *$ & $.001 * *$ & $.001 * *$ \\
\hline
\end{tabular}


Table 1.4 Research Question 1 Multinomial Logistic Regression Results

\begin{tabular}{|c|c|c|c|c|c|c|c|c|}
\hline & \multicolumn{4}{|c|}{ Low vs. medium coparenting } & \multicolumn{4}{|c|}{ Low vs. high coparenting } \\
\hline & RRR & $\mathrm{SE}$ & Prob & 95\% Conf Int & RRR & $\mathrm{SE}$ & Prob & $95 \%$ Conf Int \\
\hline \multicolumn{9}{|l|}{ Married (reference) } \\
\hline Rom Cohab & .308 & .171 & $.034^{*}$ & $.103-.916$ & .161 & .088 & $.001 * * *$ & $.055-.467$ \\
\hline Friends & .064 & .028 & $.000 * * *$ & $.026-.152$ & .017 & .008 & $.000 * * *$ & $.007-.040$ \\
\hline Sep/Div/Wid & .060 & .026 & $.000 * * *$ & $.025-.142$ & .013 & .006 & $.000 * * *$ & $.005-.031$ \\
\hline No Relationship & .032 & .014 & $.000 * * *$ & $.013-.077$ & .008 & .004 & $.000 * * *$ & $.003-.020$ \\
\hline F Age & .999 & .014 & .995 & $.972-1.208$ & 1.012 & .014 & .87 & $.985-1.040$ \\
\hline F Poverty Level & 1.030 & .046 & .513 & $.943-1.124$ & 1.029 & .046 & .531 & $.942-1.123$ \\
\hline \multicolumn{9}{|l|}{ F Education Less than HS (reference) } \\
\hline HS or Equiv & 1.06 & .277 & .817 & $.637-1.771$ & 1.021 & .269 & .935 & $.609-1.712$ \\
\hline Some college/Tech school & 1.02 & .257 & .938 & $.622-1.671$ & .949 & .242 & .836 & $.575-1.564$ \\
\hline College/grad school & .535 & .226 & .139 & $.234-1.224$ & .551 & .231 & .156 & $.241-1.254$ \\
\hline \multicolumn{9}{|l|}{$\begin{array}{l}\text { F Race/Eth Non-Hispanic White } \\
\text { (reference) }\end{array}$} \\
\hline Non-Hispanic Black & 2.079 & .515 & $.003 * *$ & $1.280-3.379$ & 2.238 & .567 & $.001 * * *$ & $1.362-3.678$ \\
\hline Hispanic & 1.40 & .415 & .254 & $.784-2.505$ & 2.297 & .680 & $.005^{* *}$ & $1.286-4.103$ \\
\hline Other & 1.63 & .978 & .418 & $.501-5.284$ & 1.301 & .799 & .668 & $.390-4.338$ \\
\hline
\end{tabular}




\section{Results for Research Q2:}

Research question 2 focuses on fathers' coparenting and fathers' time spent with the child. A one-way ANOVA was conducted to determine if fathers' perception of coparenting was different for fathers who spent different amounts of time with their child. Subjects were classified into three groups related to time spent with the focal child: all/most of the time ( $\mathrm{n}=771)$; half/some of the time/on weekends $(\mathrm{n}=298)$; and none of the time ( $\mathrm{n}=186)$. There was a statistically significant difference between groups as determined by one-way ANOVA $(F(2,1252)=91.97, p=.000)$. A Tukey post-hoc test revealed that fathers' coparenting perceptions were statistically significant differences for all groups. Fathers who reported spending the most time with their child also reported having the highest rating in coparenting relationship with the mother. Fathers who spent some or half time with their child were the next highest and those fathers who reported spending no time with their child rated their coparenting relationship the lowest.

A multinomial logistic regression was used to predict the coparenting level of fathers in the study. Fathers' coparenting support (mean scale) was divided into three categories (low, medium, high) with low coparenting support being the reference category. The independent variable was time spent with child with "none of the time" as the reference category. Four other independent variables (education, race/ethnicity, age, and poverty level) were added as control variables with time spent categories. Results of the multinomial logistic analysis indicated that the five-predictor model provides a statistically significant prediction of coparenting support level, -2 Log Likelihood $=-$ $1115.40, X^{2}(20, N=1255)=226.82, p<.001$. 
Medium Coparenting Versus Low Coparenting by Time Spent: Fathers in the "all" time category had a 3.9 times greater relative risk of reporting medium coparenting versus low coparenting support when compared to fathers in the "none" time category. Fathers in the "half/some" time spent category showed a 1.24 times relative risk of reporting medium coparenting versus low coparenting support when compared to fathers in the "none" time spent category.

The majority of the control variables were found to not be statistically significant in the medium versus low coparenting support categories. The only exception is that Non-Hispanic Black fathers had a 1.8 times greater relative risk to report medium coparenting support versus low coparenting support when compared to Non-Hispanic White fathers.

High Coparenting Versus Low Coparenting by Time Spent: Fathers in the "all" time category had a 14.62 times greater relative risk of reporting high coparenting versus low coparenting support when compared to fathers in the "none" time category. Fathers in the "half/some" time spent category showed a 1.84 times relative risk of reporting high coparenting versus low coparenting support when compared to fathers in the "none" time spent category.

The majority of the control variables were found to not be statistically significant in the high versus low coparenting support categories. The only exceptions were minority fathers in the Non-Hispanic Black and Hispanic categories. Non-Hispanic Black fathers had a 1.8 times greater relative risk to report high coparenting support versus low coparenting support when compared to Non-Hispanic White fathers. Hispanic Fathers 
had a 2.11 times greater relative risk to report high coparenting support when compared to Non-Hispanic White fathers.

Since minority fathers were showing statistical significance in the overall model, additional interaction analysis between time spent with child and fathers' race/ethnicity were conducted. Statistical significance was found in only one group in the high coparenting versus low coparenting category. This group was the Non-Hispanic Black fathers in the "none" time spent category. This category of fathers was found to have a 5.9 greater relative risk to report high coparenting versus low coparenting support when compared to Non-Hispanic White fathers. The evidence found in research question 2 supports the hypothesis that fathers who spend more time with their child will report significantly higher coparenting support. Please refer to chart 2.1 and tables 2.1 and 2.2 below for a visual representation of results. Chart 2.1 presents the predicated probabilities of fathers' coparenting rating by time spent with the child. Table 2.1 presents the ANOVA and Tukey's post hoc results. Table 2.2 presents the results of the multinomial logistic regression which includes control variables. 


\section{Chart 2.1 Predicted Probability of Coparenting by Time Spent}

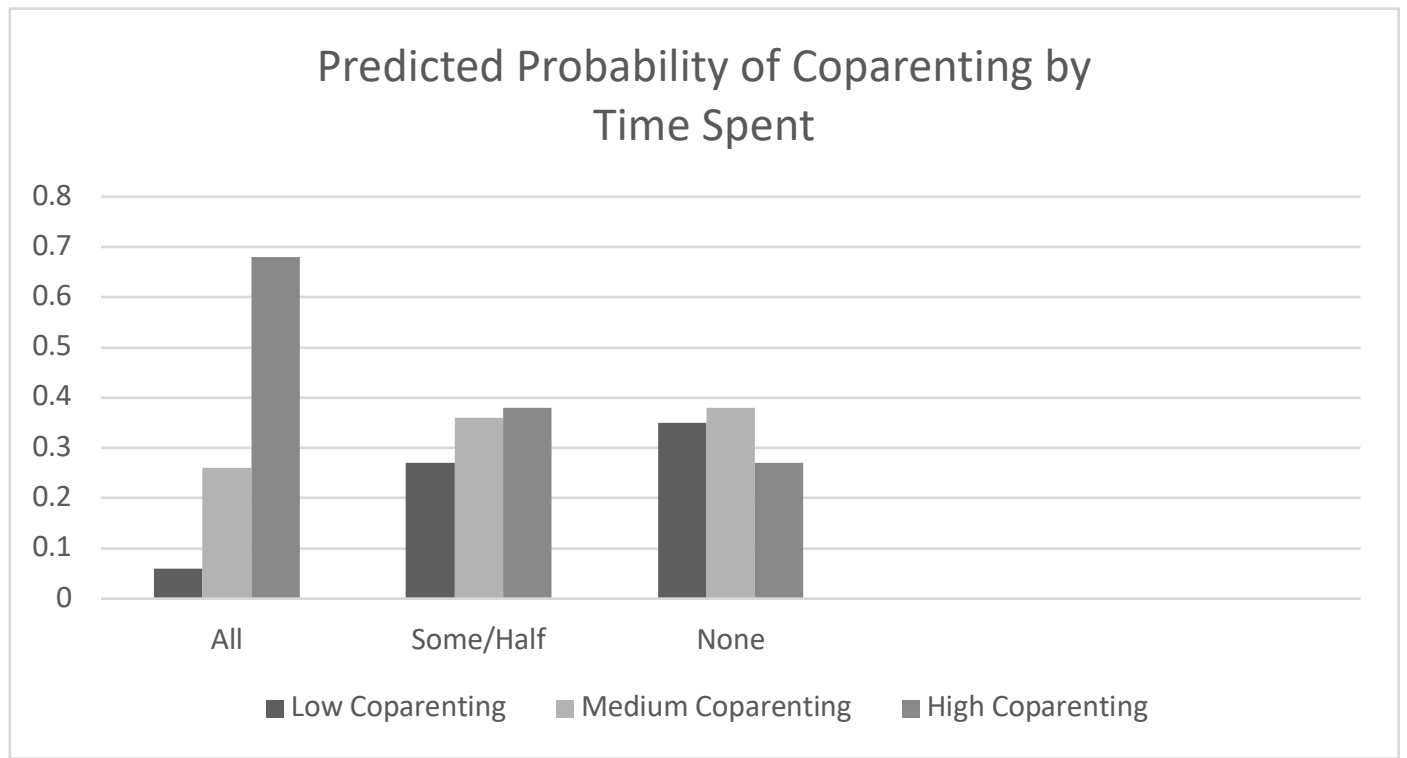

Table 2.1: Research question 2 ANOVA with Tukey post hoc results

\begin{tabular}{lccclc}
\hline & $n$ & Mean & $S D$ & \multicolumn{1}{l}{ Tukey's Comparisons } \\
\hline $\begin{array}{l}1=\text { All/most of } \\
\text { time }\end{array}$ & 771 & 3.79 & .473 & & 2 \\
$\begin{array}{l}2=\text { Half/some/ } \\
\text { weekends }\end{array}$ & 298 & 3.42 & .683 & $0.001^{* * *}$ & \\
$\begin{array}{l}3=\text { None of the } \\
\text { time }\end{array}$ & 186 & 3.21 & .819 & $0.001^{* * *}$ & $0.001^{* * *}$ \\
$* * *=p<.001$ & & & & & \\
\end{tabular}


Table 2.2 Research Q2 Multinomial Logistic Regression Results

\begin{tabular}{|c|c|c|c|c|c|c|c|c|}
\hline & \multicolumn{4}{|c|}{ Low vs. medium coparenting } & \multicolumn{4}{|c|}{ Low vs. high coparenting } \\
\hline & RRR & $S E$ & Prob & $95 \%$ Conf Int & RRR & $S E$ & Prob & $95 \%$ Conf Int \\
\hline \multicolumn{9}{|l|}{$\begin{array}{l}\text { None of the time } \\
\text { (reference) }\end{array}$} \\
\hline $\begin{array}{l}\text { Half/some of the time/ } \\
\text { weekends }\end{array}$ & 1.24 & .285 & .350 & $.790-1.946$ & 1.841 & .452 & $.013 * *$ & $1.139-2.979$ \\
\hline All of the time & 3.95 & .987 & $\begin{array}{l}.000 * * \\
*\end{array}$ & $2.416-6.441$ & 14.628 & 3.755 & $.000 * * *$ & $8.844-24.193$ \\
\hline F Age & 1.014 & .014 & .321 & $.986-1.042$ & 1.032 & .014 & $.023 *$ & $1.004-1.060$ \\
\hline F Poverty Level & 1.047 & .048 & .321 & $.956-1.15$ & 1.054 & .047 & .237 & $.966-1.151$ \\
\hline \multicolumn{9}{|l|}{$\begin{array}{l}\text { F Education Less than HS } \\
\text { (reference) }\end{array}$} \\
\hline $2 \mathrm{HS}$ or Equiv & 1.066 & .273 & .802 & $.645-1.760$ & 1.029 & .261 & .909 & $.626-1.694$ \\
\hline Some college/Tech school & 1.060 & .264 & .813 & $.651-1.726$ & 1.052 & .258 & .837 & $.650-1.701$ \\
\hline College/graduate school & .895 & .350 & .777 & $.415-1.927$ & 1.129 & .422 & .746 & $.542-2.35$ \\
\hline \multicolumn{9}{|l|}{ F Race/Eth Non-Hispanic } \\
\hline White (reference) & & & & & & & & \\
\hline Non-Hispanic Black & 1.824 & .432 & $.011 * *$ & $1.147-2.901$ & 1.814 & .421 & $.010 * *$ & $1.150-2.859$ \\
\hline Hispanic & 1.376 & .395 & .266 & $.784-2.414$ & 2.117 & .582 & $.006^{* *}$ & $1.236-3.627$ \\
\hline Other & 1.697 & .960 & .350 & $.560-5.145$ & 1.331 & .743 & .608 & $.445-3.978$ \\
\hline
\end{tabular}




\section{Results for Research Q3:}

Results for research question 3 was divided into four parts: (1) Multiple regression model for Child Reading Scores, (2) Multiple regression model for Child Math Scores, (3) Binomial logistic regression for Child Internalizing Behaviors, and (4) Binomial logistic regression for Child Externalizing Behaviors. All models included the main independent variable (father coparenting support) as well as four control variables (fathers' education level, race/ethnicity, age, and poverty level).

Please refer to Table 3.1, 3.2, 3.3, 3.4 and Chart 3 for a visual representation of the Research Question 3 analysis results. Table 3.1 presents the regression results for analysis on the child's reading scores. Table 3.2 shows the results for regression analysis for the child's math scores. Table 3.3 details the results for the binary logistic regression for the child's internalizing behaviors as demonstrated in school, and Table 3.4 presents results for the binary logistic regression for the child's externalizing behaviors at school. Chart 3 presents the predicted probability of internalizing and externalizing school behaviors by coparenting level.

\section{Multiple Regression Model for Child Reading Scores}

A multiple regression was used to predict the focal child's reading scores in the study. Fathers' coparenting support (mean scale) was divided in three categories (low, medium, high) as in the previous research questions. The main independent variable was fathers' coparenting level and six other independent variables were added as control factors (fathers' education level, race/ethnicity, time spent with child, relationship status, age, and poverty level). The multiple regression analysis results determined that the seven-predictor model was statistically significant for predicting the focal child's reading 
score, $F(16,1238)=10.83, p<.001$ and accounted for approximately $12 \%$ of the variance of the child's reading scores $(R 2=.123$, Adjusted $R 2=.111)$.

However, not supporting the hypothesis, results show that fathers' coparenting levels were not significantly related to the child's reading scores. Several control variables that were found to be statistically significant predictors. Fathers' education level was found to be a predictive factor with increasing levels of education having a predictive positive increase in reading scores. Fathers with a completed high school degree or equivalent had children with an average of 3.03 points higher in reading scores. Fathers with some completed college or trade school had children with 4.97 average higher reading scores. Fathers with a completed college degree or graduate school had children with an average increase of 6.81 in reading scores.

Fathers in the minority categories of Non-Hispanic Black and Hispanic were associated with a statistically significant decrease in reading scores. Non-Hispanic Black fathers were associated with children scoring 3.42 points lower in reading scores and Hispanic fathers were associated with children scoring 4.12 points lower in reading scores. The relationship status with the child's mother was also found to be a predictor in the model. Fathers in the romantic cohabitating group were associated with their child scoring 3.32 points lower in reading scores. Fathers in the friend category with the child's mother were associated with a 4.66 point lower reading score. Lastly, the fathers' income to poverty level was found to be a statistically significant predictor with each increase in income to poverty level being associated with a .43 -point increase in reading scores. Visual results from this part of the analysis are found below in Table 3.1. 
Since minority fathers were showing statistical significance in the model, additional interaction analysis between father coparenting level and father race/ethnicity was conducted. There was no statistical significance found when investigating this interaction. 
Table 3.1 Summary of OLS Regression Analysis for Variables Predicting Child's Reading Scores at age 9

\begin{tabular}{|c|c|c|c|c|c|}
\hline Variable & $\beta$ & SE $\beta$ & t stat & Prob. & $95 \%$ Confidence Interval \\
\hline \multicolumn{6}{|c|}{ Father Coparenting Low (reference) } \\
\hline Father Coparenting Medium & -437 & 1.234 & -.35 & .724 & $-2.86-1.985$ \\
\hline Father Coparenting High & .378 & 1.25 & .30 & .762 & $-2.07-2.824$ \\
\hline F Age & -.010 & .055 & -.18 & .855 & $-.118-.098$ \\
\hline F Poverty Level & .429 & .167 & 2.56 & $.010^{* *}$ & $.101-.758$ \\
\hline \multicolumn{6}{|l|}{ F Education } \\
\hline \multicolumn{6}{|l|}{ Less than HS (reference) } \\
\hline HS or Equivalent & 3.027 & 1.106 & 2.74 & $.006 * *$ & $.858-5.196$ \\
\hline Some college or trade school & 4.97 & 1.061 & 4.69 & $.000 * * *$ & $2.891-7.055$ \\
\hline College or graduate school & 6.806 & 1.510 & 4.51 & $.000 * * *$ & $3.843-9.770$ \\
\hline \multicolumn{6}{|l|}{ F Race/Ethnicity } \\
\hline \multicolumn{6}{|l|}{ Non-Hispanic White (reference) } \\
\hline Non-Hispanic Black & -3.42 & 1.001 & -3.42 & $.001 * * *$ & $-5.385--1.457$ \\
\hline Hispanic & -4.124 & 1.154 & -3.57 & $.000 * * *$ & $-6.389--1.859$ \\
\hline Other & .607 & 2.080 & .29 & .770 & $-3.474-4.688$ \\
\hline \multicolumn{6}{|l|}{ F Time Spent w/Child } \\
\hline \multicolumn{6}{|l|}{ All/Most (reference) } \\
\hline Half/Some & .818 & 1.480 & .55 & .581 & $-2.086-3.722$ \\
\hline None & -.456 & 1.606 & -.28 & .776 & $-3.606-2.694$ \\
\hline \multicolumn{6}{|l|}{ F Relationship Status } \\
\hline \multicolumn{6}{|l|}{ Married (reference) } \\
\hline Romantic Cohabitating & -3.316 & 1.248 & -2.66 & $.008 * *$ & $-5.764--.867$ \\
\hline Friends & -4.660 & 1.628 & -2.86 & $.004 * *$ & $-7.854--1.465$ \\
\hline Separated/Widowed/Divorced & -2.725 & 1.795 & -1.52 & .129 & $-6.246-.795$ \\
\hline No Relationship & -2.513 & 1.811 & -1.39 & .166 & $-6.067-1.040$ \\
\hline
\end{tabular}

Note* $=p<.05 ; * *=p<.01 ; * * *=p<.001$ 


\section{Multiple Regression Model for Child Math Scores}

A multiple regression was also used to predict the focal child's math scores using a standardized math test. Fathers' coparenting support (mean scale) was divided in three categories (low, medium, high) as in the previous research questions. The main independent variable was fathers' coparenting level as well as six other independent variables added as control factors (fathers' education level, race/ethnicity, time spent with child, relationship status, age, and poverty level). The multiple regression analysis results determined that the five-predictor model was statistically significant for predicting the focal child's math score, $F(16,1238)=10.71, p<.001$ and accounted for approximately $12 \%$ of the variance of the child's reading scores $(R 2=.122$, Adjusted $R 2=.110)$.

One of the fathers' coparenting levels were found to be statistically significant related to their child's math scores. Fathers' rating their coparenting support as "medium" when compared to those rating coparenting as "low" had children who had 2.99 points higher math scores, on average. Fathers' rating their coparenting support as "high" (vs. low) were not found to be significant.

There were several control variables that were found to be at the level of statistical significance. The fathers' education level was found to be a predictive factor with increasing levels of education having a predictive positive increase in math scores. Fathers with some completed college or trade school were associated with an increase of 4.55 points in math scores, and those fathers with a completed college degree or graduate school were associated with an increase of 6.94 points in math scores. Fathers in the minority categories of Non-Hispanic Black and Hispanic were associated with statistically significant decreases in math scores. Non-Hispanic Black fathers were 
associated with children scoring a 4.90 point decrease in math scores, and Hispanic fathers were associated with children having a 3.22 point decrease in math scores. Fathers' relationship status with the child's mother was found to be significant predictor. Fathers in the romantic cohabitating relationship were associated with children having 3.91 points lower on math scores. Fathers' in the friend category were associated with 5.29 points lower math scores, and fathers in the separated/divorced/widowed category were associated with 4.38 points lower on math scores. Lastly, the fathers' income to poverty level was found to be a statically significant predictor with each unit increase in income being associated with a .67 unit increase in math scores. Please refer to Table 3.2 for a visual representation of the analysis results.

Once again, minority fathers were showing statistical significance in the model, additional interaction analysis was conducted between father coparenting level and father race/ethnicity. There was no statistical significance found when investigating this interaction. 
Table 3.2 Summary of OLS Regression Analysis for Variables Predicting Child's Math Scores at age 9

\begin{tabular}{|c|c|c|c|c|c|}
\hline Variable & $\beta$ & SE $\beta$ & t stat & Prob & $95 \%$ Confidence Interval \\
\hline \multicolumn{6}{|c|}{ Father Coparenting Low (reference) } \\
\hline Father Coparenting Medium & 2.99 & 1.481 & 2.02 & $.043 *$ & $-.088-5.902$ \\
\hline Father Coparenting High & 1.899 & 1.496 & 1.27 & .205 & $-1.037-4.835$ \\
\hline F Age & .075 & .066 & 1.14 & .255 & $-.054-.205$ \\
\hline F Poverty Level & .674 & .201 & 3.35 & $.001 * * *$ & $.279-1.068$ \\
\hline \multicolumn{6}{|c|}{ F Education Less than HS (reference) } \\
\hline HS or Equivalent & 2.292 & 1.327 & 1.73 & .084 & $-.311-4.896$ \\
\hline Some college or trade school & 4.552 & 1.274 & 3.57 & $.000 * * *$ & $2.053-7.051$ \\
\hline College or grad school & 6.943 & 1.813 & 3.83 & $.000 * * *$ & $3.386-10.500$ \\
\hline \multicolumn{6}{|c|}{$\begin{array}{l}\text { F Race/Ethnicity Non-Hispanic White } \\
\text { (reference) }\end{array}$} \\
\hline Non-Hispanic Black & -4.902 & 1.201 & -4.08 & $.000 * * *$ & $-7.260--2.544$ \\
\hline Hispanic & -3.221 & 1.385 & -2.32 & $.020 * *$ & $-5.940--.503$ \\
\hline Other & .583 & 2.497 & .23 & .815 & $-4.316-5.482$ \\
\hline \multicolumn{6}{|l|}{ F Time Spent w/Child } \\
\hline \multicolumn{6}{|l|}{ All/Most (reference) } \\
\hline Half/Some & 2.587 & 1.777 & 1.46 & .146 & $-.898-6.073$ \\
\hline None & .807 & 1.927 & .42 & .676 & $-2.974-4.587$ \\
\hline \multicolumn{6}{|l|}{ F Relationship Status } \\
\hline \multicolumn{6}{|l|}{ Married (reference) } \\
\hline Romantic Cohabitating & -3.908 & 1.498 & -2.61 & $.009 * *$ & $-6.848--.969$ \\
\hline Friends & -5.286 & 1.954 & -2.70 & $.007 * *$ & $-9.121--1.451$ \\
\hline Separated/Widowed/Divorced & -4.384 & 2.154 & -2.04 & $.042 *$ & $-8.610--.158$ \\
\hline No Relationship & -3.248 & 2.174 & -1.49 & .136 & $-7.513-1.018$ \\
\hline
\end{tabular}

Note. $* * *=p<.001 \quad * *=p<.01 \quad *=p<.05$ 


\section{Binary Logistic Regression Model for Child Internalizing Behaviors}

The next step of the analysis focused on the child's internalizing behaviors demonstrated at school. A binary logistic regression was used to model the binary variable of exhibiting internalizing behaviors at school (using "not exhibiting" as the reference category). The dependent variable for this part of research question 3 is a binary level variable with 0 coded as not exhibiting internalizing skills and 1 coded as exhibiting internalizing skills. Fathers' coparenting support (mean scale) was divided in three categories (low, medium, high) as in the previous research questions. The main independent variable was fathers' coparenting level as well as six other independent variables added as control factors (fathers' education level, race/ethnicity, time spent with child, relationship status, age, and poverty level). The overall seven-predictor model provided a statistically significant prediction for the child's exhibiting internalizing behaviors $\chi^{2}(16, N=1255)=38.04, p<.001$. However, only two of the control variables (fathers in the friend relationship category and fathers' income to poverty ratio) were found to be statistically significant. Fathers who claimed to be just friends with the child's mother had a 1.69 greater relative risk for exhibiting internalizing behaviors at school. For every unit increase in the father's income, there was $10 \%$ lower odds for the child to exhibit internalizing behaviors at school. 
Table 3.3 Summary of Binary Logistic Regression Analysis for Variables Predicting Child's Internalizing Behaviors at age 9

\begin{tabular}{|c|c|c|c|c|c|}
\hline Variable & Odds Ratio & SE $\beta$ & z stat & Prob. & 95\% Confidence Interval \\
\hline \multicolumn{6}{|c|}{ Father Coparenting Low (reference) } \\
\hline Father Coparenting Medium & .972 & .185 & -.15 & .884 & $.670-1.412$ \\
\hline Father Coparenting High & .958 & .184 & -.22 & .826 & $.658-1.397$ \\
\hline F Age & 1.014 & .009 & 1.68 & .094 & $.997-1.032$ \\
\hline F Poverty Level & .903 & .026 & -3.47 & $.001 * * *$ & $.851-.956$ \\
\hline \multicolumn{6}{|l|}{ F Education } \\
\hline \multicolumn{6}{|l|}{ Less than HS (reference) } \\
\hline HS or Equivalent & .993 & .169 & -.04 & .966 & $.711-1.39$ \\
\hline Some college or trade school & .954 & .156 & -.29 & .773 & $.693-1.314$ \\
\hline College or grad school & .893 & .208 & -.48 & .629 & $.565-1.411$ \\
\hline \multicolumn{6}{|l|}{ F Race/Ethnicity } \\
\hline \multicolumn{6}{|l|}{ Non-Hispanic White (reference) } \\
\hline Non-Hispanic Black & 1.087 & .168 & .54 & .588 & $.804-1.471$ \\
\hline Hispanic & .893 & .158 & -.64 & .523 & $.629-1.265$ \\
\hline Other & 1.337 & .430 & .90 & .367 & $.712-2.510$ \\
\hline \multicolumn{6}{|l|}{ F Time Spent w/Child } \\
\hline \multicolumn{6}{|l|}{ All/Most (reference) } \\
\hline Half/Some & .850 & .196 & -.71 & .479 & $.541-1.334$ \\
\hline None & .759 & .189 & -1.11 & .268 & $.466-1.237$ \\
\hline \multicolumn{6}{|l|}{ F Relationship Status } \\
\hline \multicolumn{6}{|l|}{ Married (reference) } \\
\hline Romantic Cohabitating & 1.278 & .245 & 1.28 & .201 & $.877-1.860$ \\
\hline Friends & 1.690 & .426 & 2.07 & $.039 *$ & $1.027-2.767$ \\
\hline Separated/Widowed/Divorced & 1.340 & .387 & 1.21 & .226 & $.813-2.405$ \\
\hline No Relationship & 1.631 & .457 & 1.75 & .081 & $.942-2.826$ \\
\hline
\end{tabular}

Note. $* * *=p<.001 \quad * *=p<.01 \quad *=p<.05$ 


\section{Binary Logistic Regression Model for Child Externalizing Social Skills}

A binary logistic regression was used to model the binary variable of exhibiting externalizing behaviors (using "not exhibiting" as the reference category). The dependent variable for this part of research question 3 is a binary level variable with 0 coded as not exhibiting externalizing behaviors and 1 coded as exhibiting externalizing behaviors. Fathers' coparenting support (mean scale) was divided in three categories (low, medium, high) as in the previous research questions. The main independent variable was fathers' coparenting level as well as six other independent variables added as control factors (fathers' education level, race/ethnicity, time spent with child, relationship status, age, and poverty level). The overall seven-predictor model provided a statistically significant prediction for the child's exhibiting externalizing behaviors $\chi 2(16, N=1255)=142.38, p$ $<.001$.

Fathers' coparenting support was not found to be statistically significantly related to child externalizing disorders. Non-Hispanic Black fathers were found to be a statistically significant at predicting the odds for exhibiting externalizing behavior at school with 1.47 times greater odds for exhibiting externalizing behaviors. Hispanic fathers showed 33\% lower odds of having their child exhibit externalizing behaviors at school. A fathers' relationship status was found to be a significant predictor of externalizing behaviors at school. Fathers in the romantic cohabitating category had 1.54 times greater odds of externalizing behaviors. Fathers in the friend category had 3.1 times greater odds of having externalizing behaviors. Fathers in the separated/widowed/divorced category were at 1.79 greater odds of having their child exhibit externalizing behaviors, and fathers in the no relationship category showed 2.66 
greater odds of having children who exhibited externalizing behaviors at school. Fathers' income was also found to be a significant predictor of the odds for externalizing behavior. For every unit of increase in income, fathers were showed $6 \%$ lower odds in their child exhibiting externalizing behaviors. 
Table 3.4 Summary of Binary Logistic Regression Analysis for Variables Predicting Child's Externalizing Behaviors at age 9

\begin{tabular}{|c|c|c|c|c|c|}
\hline Variable & Odds Ratio & $\mathrm{SE} \beta$ & z stat & Prob. & $95 \%$ Confidence Interval \\
\hline \multicolumn{6}{|c|}{ Father Coparenting Low (reference) } \\
\hline Father Coparenting Medium & .816 & .160 & -1.04 & .301 & $.556-1.120$ \\
\hline Father Coparenting High & .704 & .139 & -1.78 & .076 & $.478-1.037$ \\
\hline F Age & 1.002 & .009 & .20 & .843 & $.984-1.019$ \\
\hline F Poverty Level & .941 & .029 & -1.93 & $.054 *$ & $.885-1.000$ \\
\hline \multicolumn{6}{|c|}{ F Education Less than HS (reference) } \\
\hline HS or Equivalent & 1.015 & .179 & .08 & .935 & $.719-1.433$ \\
\hline Some college or trade school & .785 & .134 & -1.42 & .156 & $.562-1.097$ \\
\hline College or grad school & .621 & .159 & -1.87 & .062 & $.376-1.024$ \\
\hline \multicolumn{6}{|c|}{ F Race/Ethnicity Non-Hispanic White } \\
\hline Non-Hispanic Black & 1.470 & .239 & 2.37 & $.018 *$ & $1.069-2.022$ \\
\hline Hispanic & 672 & .131 & -2.05 & $.041 *$ & $.459-.984$ \\
\hline Other & .787 & .288 & -.66 & .512 & $.384-1.613$ \\
\hline \multicolumn{6}{|l|}{ F Time Spent w/Child } \\
\hline Half/Some & .709 & .168 & -1.45 & .147 & $.446-1.128$ \\
\hline None & .919 & .235 & -.33 & .744 & $.557-1.519$ \\
\hline \multicolumn{6}{|l|}{ F Relationship Status Married } \\
\hline Romantic Cohabitating & 1.543 & .309 & 2.17 & $.030 *$ & $1.043-2.285$ \\
\hline Friends & 3.096 & .807 & 4.34 & $.000 * * *$ & $1.857-5.159$ \\
\hline Separated/Widowed/Divorced & 1.790 & .514 & 2.03 & $.043 *$ & $1.019-3.140$ \\
\hline No Relationship & 2.660 & .767 & 3.39 & $.001 * * *$ & $1.511-4.679$ \\
\hline
\end{tabular}




\section{Chart 3.1 Predicted Probability of Internalizing \& Externalizing Behaviors by Coparenting Levels}

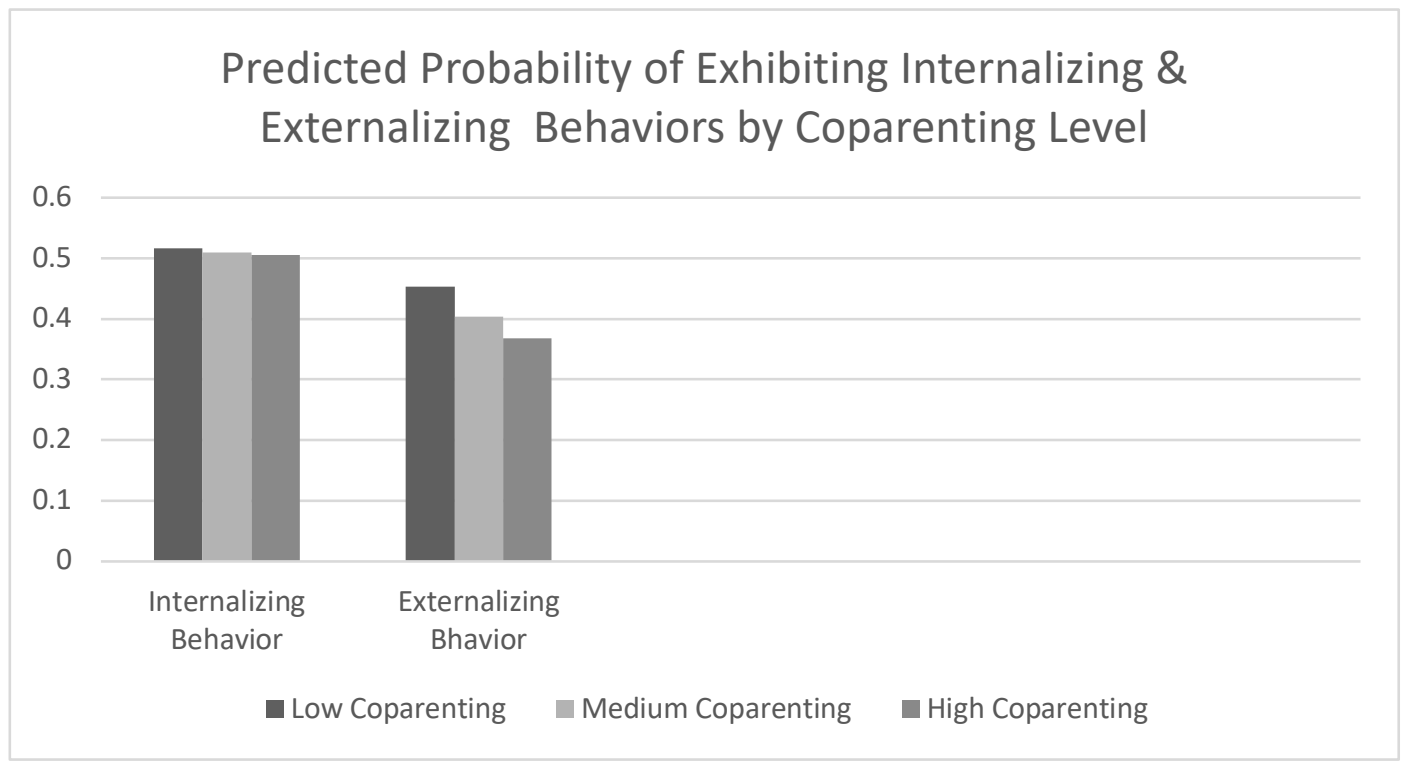

In research question 3, there were mixed findings for support of the original research hypotheses. With regards to the focal child's reading scores, evidence did not support the hypothesis that higher levels of coparenting support predicted the likelihood of having higher reading scores. While investigating child's math scores, results did partially support the hypothesis in that fathers with medium coparenting support levels (compared to low coparenting support) predicted higher math scores. Results for the investigation of child's internalizing and externalizing behaviors did not support the hypothesis that coparenting support predicted these behaviors. 


\section{CHAPTER 5: DISCUSSION}

Chapter 5 includes a summary of the research questions and hypothesis that guided this research, a summary of the results from the data analysis including connections to previous research related to the results, implications that this research brings to academic study as well as social work practice, limitations of this study, and recommendations for future research areas.

\section{Purpose of the Study:}

Working from an ecological family systems theory and using the additional lenses of Feinberg's Model of Coparenting, Emotional Security Theory, and the Family Stress Model, the current study contributes to research by exploring the relationship between fathers' perception of coparenting support and school success (academic and behavioral) when the child was age 9 controlling for fathers' race/ethnicity, age, level of education and poverty. This study also explored the relationship of fathers' coparenting support and time spent with the child as well as the status of the relationship with the mother in an effort to replicate earlier findings on the coparenting relationship based on mothers' reports of coparenting. Three main hypotheses were explored and results from each are discussed below:

- Research Question 1: Are there differences in fathers' perceptions of coparenting by relationship status (with child's mother) and are these differences significant when controlling for background factors (education, poverty level, age, race/ethnicity)?

- Hypothesis 1: Fathers who are married or cohabitating with their child's mother will report higher coparenting support levels. 
- Research Question 2: Are there differences in fathers' perceptions of coparenting by time spent with the child and are these differences significant when controlling for background factors (education, poverty level, age, race/ethnicity)?

- Hypothesis 2: Fathers who spend more time with their child will report higher coparenting support levels.

- Research Question 3: Are fathers' perceptions of coparenting associated with the focal child's school success (academic and behavioral measures) at age 9 and are these differences significant when controlling for background factors (education, poverty level, age, race/ethnicity)?

- Hypothesis 3: Fathers reporting a higher level of coparenting relationship with the child's mother will have children who are finding higher success in school as measured by standardized academic tests (higher reading and math scores) and behavior measures (lower rates of displayed externalizing and internalizing behaviors at school).

\section{Research Hypothesis 1}

Research Hypothesis 1 proposed that there would be differences in fathers' perceived coparenting support by their relationship status in that married or cohabitating fathers would report higher coparenting. Results found this hypothesis to be supported. Fathers in both the married and cohabiting relationship status categories had significantly higher ratings of coparenting when compared to the other relationship group categories. The other groups who did not have regular physical contact with their child (friends, separated/divorced/widowed, and the no relationship group) reported significantly lower coparenting. Fathers in the "no relationship" category rated their coparenting as the lowest, and this was found to be significantly different when compared to all other categories.

After dividing the coparenting ratings into three distinct groups (low, medium, high) and running multinomial logistic regression analysis, this hypothesis continued to be supported. With married fathers as the reference category, cohabitating fathers had the 
lowest relative risk of reporting medium or low coparenting, when compared against high coparenting. Fathers who described themselves in the friends or separated/divorced/widowed group had the next highest relative risk, and fathers in the "no relationship" group had the highest relative risk for reporting medium or low coparenting.

These findings suggest that fathers who co-reside with their child's mother tend to experience higher levels of coparenting support. In a Family Systems Theory view, this greater physical proximity and higher coparenting support will then lend positive benefits to the family system as a whole. When the couple is co-residing (married, romantic cohabitating), then there is a greater chance of other parts of the relationship, like coparenting, being able to function in a healthy way. Perhaps this is simply the physical proximity of the parents so they can work together as issues arise. As relationships get away from co-residency (friends, separated/widowed/ divorced, or no relationship), the coparenting sub-system seems to suffer. Additionally, when mothers and fathers are not married or living together, there is a chance that the parents have re-partnered in another relationship which adds a level of complexity to the parenting system.

These findings are consistent to recent studies on relationship status and coparenting (e.g. Carlson \& Hognas, 2011; Goldberg \& Carlson, 2015; and Waller, 2012). Past studies have revealed that positive coparenting may be more challenging to couples when unmarried parents separate or were never co-residing (Waller, 2012). When families are co-residing, positive coparenting interactions strengthen the relationship. However, when parents are not co-residing, coparenting may be the only level of interaction they have with one another. If that one level of interaction gets 
strained, then the entire coparenting relationship can be at risk (Carlson \& Hognas, 2011).

Of course, there is also the topic of new relationship partners when parents are not coresiding. While this specific study did not isolate the variable of fathers' or mothers' repartnering, past researchers have noted that new partners in either parent's life can negatively affect the coparenting relationship (Bronte-Tinkew \& Horowitz, 2010; Kamp Dush et.al., 2011).

The results from Research Question 1 also support the Family Systems Theory in general in that when the father/mother relationship is more intact (married, romantic cohabitating) then the coparenting sub-system is rated higher. Other researchers have found that married and romantically cohabitating parents have similar outcomes when rating coparenting (Cabrera et. al., 2012; Goldberg \& Carlson, 2015).

\section{Research Hypothesis 2}

This hypothesis explored the relationship between how much time a child spends with his/her biological father and the father's coparenting support. It was hypothesized that fathers who spent more time with their child would rate their coparenting support higher. Fathers were grouped into three categories of time spent with their child: (1) all/most of the time, (2) half/some of the time, and (3) none of the time. There was a statistically significant difference found between the groups as hypothesized. Results indicated that fathers who spent more time with their child rated their coparenting support as higher. Additionally, when multivariate analysis was run using multinomial logistic regression with three distinct coparenting groups (low, medium, high), it also supported the hypothesis that fathers who spent more time with their child were more likely to rate their coparenting higher. 
The findings for Research Question/Hypothesis 2 suggest that the more relational time the father spends with the child, the more positive the father will rate his coparenting support. This could be explained by the fact that as the father spends more time and is therefore more involved, it creates an investment in the relationship with the child's mother which in turn builds trust in the coparenting relationship. This investment of parenting involvement of the father then leads to the father feeling increased coparenting support from the mother and creates a positive feedback loop in the coparenting relationship. This suggests that time with the child is investment in the father-child relationship, and this promotes a feeling of positive coparenting support.

This study's findings are consistent in supporting a Family Systems Theory view. A Family Systems Theory view poses that when fathers spend more time with their child, they are creating a solid foundation for the coparenting sub-system with the mother and vice-versa creating a feedback loop (Minuchin, 1974). This in turn strengthens the coparenting sub-system which positively affects other parts of the family system. Also, within Family Systems Theory, the concept of spillover can be supported (Crouter,1984). As the father spends more time with the child, the father-child sub-system positively influences the father's perception of the coparenting sub-system. While this research study was a parsimonious study to explore coparenting and child outcomes using data points other than those expressed by mothers and, therefore, can only suggest possible links to the theory, future studies could investigate mediating and moderating family systems mechanisms to further test the theory.

Other family researchers have also made mention of how the amount of time spent between child and father has positive or negative impacts on the coparenting 
relationship (Carlson et. al., 2008; Perry et al, 2012; Waller, 2012). Perry and colleagues (2012) found that fathers in unmarried, cohabitating relationships who received higher levels of support from the mother were more actively involved with their child indicating that it may be the support that encourages the father to spend more time with the child which in turn makes investments in the child's life and the family system. Likewise, Waller (2012) found that fathers who reported conflicted coparenting with the mother spent less time with the child. Fathers were significantly more likely to spend time with their child when they had a positive view of coparenting (Waller, 2012). Carlson and colleagues (2008) report that when a father feels coparenting support from the mother, he is more likely to then spend time with the child.

To be noted in Research Question 2, there was a significant interaction between fathers' race and coparenting rating with one group. After additional interaction analysis, it was found that Non-Hispanic Black fathers had a significantly higher relative risk (5.9 times) to report high levels of coparenting (versus low coparenting) when compared to Non-Hispanic White fathers, who were used as the reference category. Since this finding is specific to this particular ethnic group, it suggests that something within the cultural context of Non-Hispanic Black family systems explain this dynamic.

To offer a possible explanation for this finding, Edin and Nelson (2014), in their book of over a hundred interviews with low-income minority fathers in Philadelphia, found that fathers who persist through many financial and personal barriers to maintain their relationship with their child and child's mother describe themselves as doing very well as a parent, especially if they had been abandoned by their own father as a child. Other researchers have also made note of this ethnic and cultural difference and have 
surmised that historically Non-Hispanic Black family structures are more likely to be in non-married cohabitating and non-cohabitating relationships, and fathers still manage to stay connected to and spend time with their child (Mincy \& Pouncy, 2007; Perry et. al., 2012; Shannon, Cabrera, Tamis-LeMonda, \& Lamb, 2009). Shannon and colleagues (2009) specifically found that while the unions in Non-Hispanic Black families tend to be more unstable as defined by traditional research categories, the amount of time the fathers' spent on a monthly basis with the child was the indicator of relationship quality with the child, rather than type of residency with the mother.

\section{Research Hypothesis 3}

The final hypothesis for the study was that fathers who rate their coparenting relationship higher will have children who exhibit higher academic test scores (higher reading and math scores) and lower behavioral ratings (lower rates of externalizing and internalizing behaviors) at school. This question had four parts: examining the relationship of coparenting with standardized test (1) reading scores and (2) math scores as well as examining the relationship of coparenting with (3) externalizing school behaviors and (4) internalizing school behaviors.

Evidence was not found in the analysis to support the hypothesis that fathers who rate their coparenting higher predicted higher reading scores. However, several of the control variables were found to be significant predictors. Fathers who had higher levels of education predicted higher levels of reading scores at statistically significant levels. Each level of education (high school, some college/trade school, and college) predicted increasingly higher reading scores. Fathers in the minority categories of Non-Hispanic Black and Hispanic were associated with predicting lower reading scores. The 
relationship status of romantic cohabitating and friends with their child's mother predicted significantly lower reading scores. Additionally, fathers' income to poverty level was found to be a significant predictor of reading scores with increases in income being associated with higher reading scores.

The study hypothesis was partially supported for a relationship between fathers' coparenting and math scores. Fathers rating their coparenting as medium when compared to low ratings predicted significantly higher math scores. However, fathers in the high coparenting group when compared to the low coparenting group did not predict higher math scores. Several control variables also contributed explanations to the model. Fathers' education level was once again found to be a significant predicting factor with increasing levels of education predicting higher math scores. Race/ethnicity was also found to be significant predictors with Non-Hispanic Black and Hispanic fathers associated with predicting lower reading scores. The fathers' poverty level was also found to be a significant predictor with each unit of income being associated with an increase in math scores. Fathers' relationship status with the child's mother was also found to be a significant predictor of decreased math scores. Fathers in the categories of romantic cohabitating, friends, and separated/widowed/divorced predicted increasingly lower math scores for each category respectively. Other researchers have found similar results. Stight and Neitzel (2003) had found that when parents of third graders who had poor coparenting relations were associated with lower grades and poorer social adjustment in school.

The analysis for the child's internalizing school behaviors did not support the original study hypothesis. While the overall seven-predictor model was found to 
significantly predict child's internalizing behaviors, only fathers' income to poverty ratio and the relationship category of friends were found to be a statistically significant. For every unit of increase in the fathers' income, there was $10 \%$ lower odds of the child showing internalizing behaviors in school. Fathers who reported being just friends with the child's mother saw a 1.69 greater relative risk for showing internalizing behaviors in school.

Lastly, there was not support for the last study hypothesis involving the child's externalizing behaviors at school. Fathers coparenting support was not found to be a statistically significant predictor. Several of the control variables were found to be significant predictors. Race/ethnicity offered an explanation to this hypothesis with NonHispanic Black fathers predicting higher rates of externalizing behaviors at a significant level and Hispanic fathers showing lower odds of having their child exhibit externalizing behaviors. In a study by Lindahl and Malik (1999), they found significant difference in parenting styles by ethnicity. Significant parenting style differences were found when comparing Hispanic families and Non-Hispanic White families. Hispanic families who used a hierarchical style of parenting reported less externalizing child behaviors while Non-Hispanic White families saw an increase in externalizing child behaviors when using the hierarchical parenting style. There could be a cultural factor explaining results in the current study to be explored in a future study.

Fathers' income was also a significant finding with increased income predicting a decrease for displaying externalizing behaviors at school. Lastly, fathers' relationship to the mother was found to be a consistent predictor for the child exhibiting externalizing behaviors at school. All other categories (romantic cohabitating, friends, 
separated/widowed/divorced, and no relationship) when compared to married parents found an increase chance for the child to exhibit externalizing behaviors at school.

These findings suggest that while fathers' coparenting support may be a contributing component to a child's school success (measured by academic tests and behavioral reports) there are other factors that seem to contribute more consistently. Namely, these factors are the fathers' race/ethnicity, his income level, and his relationship with the mother. Additionally, for both academic areas (reading and math), the higher the education level of the father predicted and increase scores.

Generally speaking, a couple of consistent predictors emerged across all three research questions. Support for the Family Stress Model was found in that the poverty level of the fathers was found to be a significant predictor in all parts of this study. As the fathers' economic security increased, the child's reading and math scores increased, and their chance of exhibiting internalizing and externalizing behaviors at school decreased. This study's findings are consistent with Goldberg and Carlson (2015) who also found higher socioeconomic levels to be important antecedents to the fathers' positive coparenting relationship. In their meta-analysis of coparenting and child outcomes, Teubert and Pinquart (2010) found evidence that the financial status of the parents was a common factor across studies in coparenting in which financial stress led to lower coparenting which in turn was associated with poor child outcomes at school.

Another important finding from this study was the significance of the relationship status between the father and mother. In all parts of Research Question 3, the relationship status was found to be a significant predictor to the child's success or challenges at school. When compared to married couples, children with parents who were romantically 
cohabitating or just friends predicted significantly lower reading and math scores. Only parents describing themselves as friends increased the chance of the child experiencing internalizing behaviors at school. However, all relationship categories, when compared to married, predicted significantly higher externalizing behavior issues at school. These findings are consistent with other studies which found that the relationship status between mom and dad had a significant impact on child outcomes (Bronte-Tinkew et. al, 2009; Fomby \& Cherlin, 2007; Fomby \& Osborne, 2010; Foster \& Kalil, 2007).

\section{Implications for Social Work Practice}

There are several implications for social work practice and program development based on the findings of this study. Social workers are called upon to serve as advocates. In light of this study's findings, social workers are encouraged to advocate for fathers to be involved whether they are married or not or living with the child's mother or not. This means not only encouraging fathers to take a more active role in nurturing and caregiving, but also advocating for programs and policies that can support fathers in these efforts. Programs that address both father involvement and barriers to a father's healthy parenting relationship would reap dividends to the child's development. Similarly, social workers can educate mothers on the importance of the protective factors of having fathers involved in their child's life. Mothers need to be made aware of gatekeeping behaviors that consciously or unconsciously limit the father's level of involvement in the parenting sub-system (Schoppe-Sullivan, Altenburger, Lee, Bower, Kamp Dush, 2015). Lastly, social work practitioners need to be aware of their own cultural gatekeeping beliefs as it pertains to a father's role with the child. In doing so, it is important for family service agencies to conduct father-friendliness assessments to ensure that they present as 
welcoming and receptive to fathers who may be otherwise reluctant to seek or accept services.

Findings from this study can also be used to guide interventions with families by focusing on stabilizing and strengthening the coparenting relationship. Interventions for the family coparenting sub-system might include helping to educate parents on the importance of their coparenting relationship and how to strengthen this part of their parenting relationship, whether or not the parents are married, cohabitating or other physical arrangement. Family system researchers also recommend that these coparenting interventions start as early as the prenatal period when unmarried fathers are more likely to be romantically involved with the child's mother (Fagan, 2008; Fagan \& Kaufman 2015; McLanahan et al., 2013; Panter-Brick et al., 2014). Building on the to-be mother and fathers' excitement of becoming parents is a prime time to discuss the importance of the coparenting relationship. Additionally, social workers must be sensitive in providing coparenting prevention and intervention programs to coparents who may not have a romantic relationship.

One of the strongest predictors in this study that was found throughout all research questions was fathers' poverty level. Fathers with lower economic stability, as measured by the poverty level, predicted poorer outcomes with the child in both academic and behavioral areas. In terms of social work policy, the profession needs to continue to support father involvement in more comprehensive ways than just child support enforcement. There is no doubt that a child's economic security is important. Children in mother-only homes experience poverty at a rate of $30.6 \%$ as compared to $5.3 \%$ of children in two-parent homes (DeNavas-Walt \& Proctor, 2015). However, much 
of the nation's historical and political attention has been focused on child support collection, when this is just one piece of the puzzle in helping fathers be involved and support their children. The Office of Child Support Enforcement has as its mission to include: helping children to receive reliable support payments by helping to locate absent parents, establishing legal paternity, establishing and enforcing child support orders, increasing health care coverage for children, and supporting parents by partnering with states to provide employment- seeking programs, health coparenting relationship programs, responsible fatherhood initiatives and other services to prevent and reduce family violence (Office of Child Support Enforcement, 2016). Findings from this study suggest that any policy investments made in fathers (economic security, education, relationship with mother) have the potential to pay off in positive ways with their children both academically and behaviorally. There have been recent positive reviews of father involvement programs that paired both stable employment assistance and education on the coparenting relationship with positive results (Randles, 2019). This type of investment in the family makes sense as results from this study indicate that the father's lower poverty level is interwoven as a constant variable in predicting lower coparenting support, more behavior issues at school, and lower levels of academic success.

\section{Limitations for this study}

\section{Limitations specific to coparenting construct}

When considering the results of this study, there are limitation that should be taken into account. First and most critical, is that the coparenting questions used in the FFCWS were not part of a previously validated scale of coparenting. Benefits of using an 
already validated coparenting scale include consistency in measuring the construct of coparenting, consistently breaking down the components of coparenting, and comparing to other coparenting research studies who have used established scales. There are several coparenting measures in existence. Examples include: the Quality of Coparent Communication scale (Ahrons, 1981), Parenting Alliance inventory (PAI: Abidin \& Brunner, 1995), and Daily Coparenting Scale (D-Cop: McDaniel, Teti, \& Feinberg, 2017), but the FFCWS did not use any previously validated instrument for studying the construct of coparenting.

The survey authors also chose to only ask the fathers a total of three coparenting questions in wave 5, when six items had been asked in previous years. This made it difficult to perform cross comparisons and get a full picture of the coparenting questions from wave 3 to wave 5 . It also limited the ability to do longitudinal analysis on coparenting questions since not all items were asked each wave of the study. Mothers were asked six coparenting questions in wave 3 and wave 5, but the fathers' chance to give opinions on their coparenting relationship with the mother was limited to only three items. This reduction in the number of survey items dedicated to fathers' coparenting made it difficult to connect the four different components of Feinberg's Model of Coparenting.

Another limitation with the survey item concerning the fathers' questions on coparenting has already been pointed out by Goldberg and Carlson (2015). Fathers were asked to reference the mothers' coparenting support, but mothers were asked the support questions and also asked additional questions to rate specific activities in the father's ability to coparent. For instance, mothers were asked if the child's father "respects the 
schedules and rules you make for the child" while fathers did not have a similar question to answer. This creates a limitation because fathers and mothers were not treated the same when measuring their coparenting behaviors and not given the same chance to voice opinions about their coparenting relationship and abilities. Additionally, neither mother nor father were asked any questions about joint coparenting interactions, such as coparenting conflict, which is a key component of coparenting. They were simply asked to report on perception of the other parent's coparenting abilities (mothers reporting on fathers only) or about coparenting support (mothers and fathers both reporting on each other). These inconsistencies make it difficult to compare the complex nature of the coparenting relationship.

Finally, another important limitation noted by other family researchers (Goldberg \& Carlson, 2015; Kamp Dush et. al., 2011) is that in the FFCWS, mothers were only asked the coparenting set of questions if the mother answered the question that the father had at least minimal contact with the child prior to the administration of the year 9 survey. This means that fathers who had no interaction with their child were left out of the results. This could overestimate the levels of positive coparenting support reported in this study.

\section{Common FFCWS Limitations}

There are other common limitations of this study due to the nature of self-report survey data. For the purpose of this study, the construct of coparenting was measured by fathers' self-report items. Limitations of self-report survey items include the potential for social desirability bias (Royse, 2011). This bias can occur with topics as sensitive as coparenting and raising children; parents might be apt to rate themselves higher and their 
partner at a lower level as a result of social or cultural expectations. Yet another limitation for surveys is the forced-choice options for respondents on the answer choices. This forced-choice answer option is convenient but limits the quality of the data, especially for a complex family dynamic like coparenting (McHale, 2011). Specifically, for this study, the coparenting questions were on a 4-point scale (always true, sometime true, rarely true, and never true), and the child's internalizing and externalizing behavior survey items were on a 4-point scale (very often, often, sometimes, and never).

Other limitations include that the data used for this study was cross-sectional, point in time, data. There are inherent limitations that come with cross-sectional data. This limits the ability to explore causality and cannot account for bi-directional influences (Setia, 2016). Additionally, the results of this study were limited to biological birth parents in urban cities who were married, romantic cohabitating, divorced/separated/widowed, or no relationship. Therefore, results of this study may not be applied or generalized to other family constellations (rural parents, same-sex parents, etc.).

\section{Analysis Limitations}

While it is valuable that the population of the FFCWS was historically more racially/ethnically diverse than early studies, for the purpose of this study, married fathers were overrepresented (43.35\%). This made the data used in this study skewed towards married fathers' feedback. Understandably, biological fathers who are living in the same household as their child and spending more time with their child are more likely to rate coparenting more positively. Therefore, after reviewing the frequency of responses, the decision was made to divide data into a low, middle, and high coparenting groupings for 
analysis. The teachers' data for this study were also skewed to the positive for the child's internalizing and externalizing behaviors. After reviewing the frequency of responses, the decision was made to make the measurement of these behaviors a binomial variable for analysis purposes.

Missing data is also a common limitation to research studies using large data sets. This particular study experienced missing data from attrition from one wave of the survey to the next from fathers not returning in this wave of the data collection. From baseline to year 9 (used for this study), twenty-two percent (22\%) of fathers had dropped out of the study. There were also high levels of missing data between cases involving fathers and teachers. Only, forty-one percent (41\%) of the cases involving biological fathers had also completed the educational assessments used in this study. Missing data may have skewed or reduced the power for the statistical analysis.

\section{Recommendations for Future Study}

Future studies could continue building on the current study by continuing to feature data collected from fathers so that they can offer their own views of coparenting rather than relying exclusively on mothers' responses to represent that area of parenting. In a way, historically allowing mothers to speak for both parents about the coparenting relationship amounts to institutional maternal gatekeeping. In future studies, it is recommended to have a survey tool that has parents jointly measure the construct of coparenting rather than having each parent report on the other. Additionally, it is recommended to incorporate more observational methods of measuring coparenting since survey items come with their own limitations. 
One limitation already mentioned above was the lack of questions that represented the four distinct components of Feinberg's Model of Coparenting, which is the most common construct mentioned in coparenting literature (Cabrera et. al., 2012). It was unfortunate that there was not enough father coparenting questions to represent the four components of Feinberg's model. In future studies, it is recommended that researchers make use instruments that are comprehensive enough to account for all four dimensions of Feinberg's Model which include (1) childrearing agreement/disagreement, (2) division of child-related duties, tasks, and responsibilities, (3) joint management of family interactions, and (4) coparenting support/undermining.

Lastly, previous researchers have found that children are fairly accurate in reporting and capturing their parents' level of functioning and conflict. In fact, two such studies found that children were more accurate in capturing key aspects of their own functioning and their parent functioning than the parents themselves (Cummings, DoekeMorey, \& Dukewich, 2001; Cummings, Davies, \& Simpson, 1994). With this in mind, it would be helpful to have a few questions that children can answer about their own family dynamics, specifically their parents' ability to coparent. In the FFCWS, the children were asked some questions about their family, but questions specific to coparenting functioning were left off. Future research should consider adding some reflective questions on the child survey about coparenting family dynamics.

\section{Conclusion}

This study was one of only a few using FFCWS data to explore the relationship of coparenting that included fathers' survey responses and did not rely on mothers' responses for any data points. This study extends the research literature by exploring the 
family system of fathers' coparenting and its relationship to the child's academic and behavioral school success. The work to understand the dynamics of the coparenting relationship is important as it directly affects a child's development at every stage.

While the explanation of fathers' coparenting support was significant for some parts of the research hypotheses, two other variables were found to be consistent predictors throughout all three research hypothesis. Namely, the fathers' poverty level and the relationship status with the mother were found to be significant predictors in the model. Lower levels of poverty predicted poorer academic and behavioral outcomes in children and married fathers predicted better academic and behavioral outcomes in children.

Future work at both the research and practice level should continue to give fathers a voice in reporting coparenting while also expanding and deepening the study of the separate components that make up the construct of coparenting. Additionally, national policy should continue to invest resources to help stabilize and strengthen coparenting relationships as well as stable employment opportunities for fathers. In doing so, we will be able to intervene earlier in the coparenting relationship in order to strengthen that subsystem and prevent children from under-performing at school. 


\section{REFERENCES}

Abidin, R.R., \& Brunner, J.F. (1995). Development of a parenting alliance inventory. Journal of Clinical Child Psychology, 24, 31-40.

Ahrons C.R. (1981). The continuing coparental relationship between divorced spouses. The American Journal of Orthopsychiatry, 51(3), 415-28.

Amato, P.R. (2000). The consequences of divorce for adults and children. Journal of Marriage and the Family, 62, 1269-1287.

Amato, P.R. (2001). Children of divorce in the 1990s: An update of the Amato and Keith (1991) meta-analysis. Journal of Family Psychology, 15(3), 355-370.

Amato, P.R. (2010). Research on divorce: Continuing trends and new developments. Journal of Marriage and the Family, 72, 650-666.

Behnke, A. O., MacDermid, S. M., Coltrane, S. L., Parke, R. D., Duffy, S., \& Widaman, K. F. (2008). Family cohesion in the lives of Mexican American and European American parents. Journal of Marriage and Family, 70(4), 1045-1059.

Belsky, J., \& Fearon, R. P. (2004). Exploring marriage-parenting typologies and their contextual antecedents and developmental sequelae. Development and Psychopathology, 16(3), 501-523.

Bendheim-Thoman Center for Research on Child Wellbeing. (2005). Childcare centers and inter-organizational ties in high poverty neighborhoods (Fragile Families Research Brief No. 34). Retrieved from http://www.fragilefamilies.princeton.edu/briefs/ResearchBrief34.pdf. 
Bendheim-Thoman Center for Research on Child Wellbeing. (2013). Scales documentation and question sources for the nine-year wave of the Fragile Families and Child Wellbeing Study. Princeton University, Princeton, NJ.

Bendheim-Thoman Center for Research on Child Wellbeing. (2014b). Fragile Families and Child Wellbeing documentation. Retrieved from http://www.fragilefamilies.princeton.edu/documentation.asp.

Benner, A. D., \& Kim, S. Y. (2010). Understanding Chinese American adolescents' developmental outcomes: Insights from the family stress model. Journal of Research on Adolescence, 20(1), 1-12.

Berryhill, M. B. (2017). Coparenting and parental school involvement. Child \& Youth Care Forum. Vol. 46 (2), 261-283.

Bialik, K. (2017, October 6). Americans deepest in poverty lost more ground in 2016. Retrieved from http://pewrsr.ch/2xmtLjc.

Boyd-Franklin, N. (2003). Black families in therapy: Understanding the African American experience (2nd ed.). New York: Guilford Press.

Brody, G. H., \& Flor, D. L. (1996). Coparenting, family interactions, and competence among African American youths. New Directions for Child and Adolescent Development, (74), 77-91.

Bronfenbrenner, U. (1979). The ecology of human development: Experiments by nature and design. Cambridge, Mass.: Harvard University Press.

Bronfenbrenner, U. (1990). Who cares for children? Research and Clinical Center for Child Development Annual Report, 12, 27-40. 
Brown, G. L., Schoppe-Sullivan, S. J., Mangelsdorf, S. C., \& Neff, C. (2010). Observed and reported supportive coparenting as predictors of infant-mother and infantfather attachment security. Early Child Development and Care, 180(1-2), 121137.

Brown, S. L., Manning, W. D., \& Stykes, J. B. (2015). Family structure and child wellbeing: Integrating family complexity. Journal of Marriage and Family, 77(1), 177-190.

Bronte-Tinkew, J., \& Horowitz, A. (2010). Factors associated with unmarried, nonresident fathers' perceptions of their coparenting. Journal of Family Issues, 31(1), 31-65.

Bronte-Tinkew, J., Horowitz, A., \& Scott, M. E. (2009). Fathering with multiple partners: Links to children's wellbeing in early childhood. Journal of Marriage and Family, $71,608-631$.

Buchanan, C.M. \& Heiges, K.L. (2001). When conflict continues after the marriage ends: Effects from conflict on postdivorce children. In J. H. Grych, \& F.D. Fincham, (Eds.), Interparental conflict and child development: Theory, research, and applications (pp. 337-362). Cambridge: Cambridge University Press.

Cabrera, N. J., Scott, M., Fagan, J., Steward-Streng, N., \& Chien, N. (2012). Coparenting and children's school readiness: A mediational model. Family Process, 51(3), 307-324.

Carlson, M. J., \& Furstenberg, F. F. (2006). The prevalence and correlates of multipartnered fertility among urban US parents. Journal of Marriage and Family, 68(3), 718-732. 
Carlson, M.J. \& Hognas, R.S. (2011). Coparenting in fragile families: Understanding how parents work together after a nonmarital birth. In J. P. McHale, K. M. Lindahl, (Eds.), Coparenting: A conceptual and clinical examination of family systems (pp. 81-104). Washington, DC, US: American Psychological Association.

Carlson, M., \& McLanahan, S. (2002). Fragile families, father involvement, and public policy. In N.J. Cabrera, T.S. Tamis-LeMonda,(Eds.), Handbook of father involvement: Multidisciplinary perspectives (pp.461-488). New York, NY: Routledge.

Carlson, M. J., McLanahan, S. S., \& Brooks-Gunn, J. (2008). Coparenting and nonresident fathers' involvement with young children after a nonmarital birth. Demography, 45(2), 461-488.

Cherlin, A. J., \& Seltzer, J. A. (2014). Family complexity, the family safety net, and public policy. Annals of The American Academy of Political and Social Science, 654(1), 231-239.

Child Trends Databank. (2015). Family structure. Retrieved from: https://www.childtrends.org/wpcontent/uploads/2015/03/59_Family_Structure.pdf

Choi, J., \& Becher, E. H. (2019). Supportive coparenting, parenting stress, harsh parenting, and child behavior problems in nonmarital families. Family Process, $58(2), 404-417$.

Conger, R. D., Conger, K. J., Elder, J., G. H., Lorenz, F. O., Simons, R. L., \& Whitbeck, L. B. (1992). A family process model of economic hardship and adjustment of early adolescent boys. Child Development, 63(3), 526-541. 
Conger, R. D., Conger, K. J., Elder, G. H., Lorenz, F. O., Simons, R. L., \& Whitbeck, L. B. (1993). Family economic stress and adjustment of early adolescent girls. Developmental Psychology, 29(2), 206-219.

Conger, R. D., Wallace, L. E., Sun, Y., Simons, R. L., McLoyd, V. C., \& Brody, G. H. (2002). Economic pressure in African American families: A replication and extension of the family stress model. Developmental Psychology, 38(2), 179-193.

Cowan, P. (2002). Forward. In J.P. McHale \& W. Grolnick (Eds.), Retrospect and prospect in the psychological study of families (pp. xv-xx). Mahwah, NJ: Lawrence Erlbaum.

Cox, M. J., \& Paley, B. (1997). Families as systems. Annual review of psychology, 48(1), 243-267.

Cronbach, L.J. (1951). Coefficient alpha and the internal structure of tests. Psychometrika, 16, 297-334.

Crouter, A. C. (1984). Spillover from family to work: The neglected side of the workfamily interface. Human Relations, 37(6), 425-441.

Cummings, E. M., \& Davies, P. (1994). Children and marital conflict: The impact of family dispute and resolution. New York, NY: Guilford Press.

Cummings, E. M., \& Davies, P. T. (2002). Effects of marital conflict on children: Recent advances and emerging themes in process-oriented research. Journal of child psychology and psychiatry, 43(1), 31-63.

Cummings, E. M., Davies, P. T., \& Simpson, K. S. (1994). Marital conflict, gender, and children's appraisals and coping efficacy as mediators of child adjustment. Journal of Family Psychology, 8(2), 141-149. 
Cummings, E. M., Goeke-Morey, M. C., \& Dukewich, T. L. (2001). The study of relations between marital conflict and child adjustment: Challenges and new directions for methodology. In J. H. Grych \& F. D. Fincham (Eds.), Interparental conflict and child development: Theory, research, and applications. (pp. 39-63). New York, NY: Cambridge University Press.

Cummings, E. M., Schermerhorn, A. C., Davies, P. T., Goeke-Morey, M. C., \& Cummings, J. S. (2006). Interparental Discord and Child Adjustment: Prospective Investigations of Emotional Security as an Explanatory Mechanism. Child Development, $77(1), 132-152$.

Davies, P. T., \& Cummings, E. M. (1994). Marital conflict and child adjustment: An emotional security hypothesis. Psychological Bulletin, 116(3), 387-411.

Davies, P. T., \& Cummings, E. M. (1998). Exploring children's emotional security as a mediator of the link between marital relations and child adjustment. Child Development, 69(1), 124-139.

Davies, P. T., \& Martin, M. J. (2013). The reformulation of emotional security theory: The role of children's social defense in developmental psychopathology. Development and Psychopathology, 25(4, Pt 2), 1435-1454.

DeNavas-Walt, C. \& Proctor, B.D. (2015). Income and poverty in the United States: 2014 U.S. Census Bureau, Current Population Reports, 60-252. U.S. Washington, DC: Government Printing Office.

Donnellan, M., Martin, M., Conger, K., \& Conger, R. (2013). Economic distress and poverty in families. In M.A. Fine \& F.D. Fincham, (Eds.). Handbook of family theories: A content-based approach (pp.338-355). New York: Routledge. 
Duncan, G. J., Magnuson, K., Kalil, A., \& Ziol-Guest, K. (2012). The importance of early childhood poverty. Social Indicators Research, 108(1), 87-98.

Duncan, G. J., Magnuson, K., \& Votruba-Drzal, E. (2017). Moving beyond correlations in assessing the consequences of poverty. Annual Review of Psychology, 68, 413434.

Edin, K., \& Nelson, T. (2014). Doing the best I can: Fatherhood in the inner city. Berkeley: University of California Press.

Emery, R. E. (1982). Interparental conflict and the children of discord and divorce. Psychological Bulletin, 92(2), 310-330.

Erel O, \& Burman B. (1995). Interrelatedness of marital relations and parent-child relations: a meta-analytic review. Psychological Bulletin, 118(1), 108-32.

Fagan, J. (2008). Randomized study of a prebirth coparenting intervention with adolescent and young fathers. Family Relations, 57(3), 309-323.

Fagan, J., \& Cabrera, N. (2012). Longitudinal and reciprocal associations between coparenting conflict and father engagement. Journal of Family Psychology, 26(6), 1004-1011.

Fagan, J., \& Cherson, M. (2017). Maternal Gatekeeping. Journal of Family Issues, 38(5), 633-653.

Fagan, J. \& Kaufman, R. (2015). Co-parenting relationships among low-income, unmarried parents: Perspectives of fathers in fatherhood programs. Family Court Review, 53(2), 304-316. 
Farr, R. H., \& Patterson, C. J. (2013). Coparenting among lesbian, gay, and heterosexual couples: Associations with adopted children's outcomes. Child Development, 84(4), 1226-1240.

Feinberg, M. E. (2002). Coparenting and the transition to parenthood: A framework for prevention. Clinical Child and Family Psychology Review, 5(3), 173-195.

Feinberg, M. (2003). The internal structure and ecological context of coparenting: A framework for research and intervention. Parenting: Science and Practice, 3(2), 95-131.

Feinberg, M. E., Kan, M. L., \& Hetherington, E. M. (2007). The Longitudinal influence of coparenting conflict on parental negativity and adolescent maladjustment. Journal of Marriage and Family, 69(3), 687-702.

Feinberg, M.E. \& Sakuma, K. (2011). Coparenting interventions for expecting parents. In J. P. McHale, K. M. Lindahl, (Eds.), Coparenting: A conceptual and clinical examination of family systems (pp. 191-210). Washington, DC: American Psychological Association.

Fomby, P., \& Cherlin, A. J. (2007). Family instability and child well-being. American Sociological Review, 72(2), 181-204.

Fomby, P., \& Osborne, C. (2010). The influence of union instability and union quality on children's aggressive behavior. Social Science Research, 39(6), 912-924.

Foster, E. M., \& Kalil, A. (2007). Living arrangements and children's development in low-income White, Black, and Latino families. Child Development, 78(6), 16571674. 
Furstenberg, F. F. (2014). Fifty years of family change: From consensus to complexity. Annals of the American Academy of Political and Social Science, 654(1), 12-30.

Garfinkel, I., McLanahan, S., \& Hanson, T. (1998). A patchwork portrait of nonresidential fathers. In Garfinkel, McLanahan, Meyer, \& Seltzer (Eds.), Fathers under fire: The revolution in child support enforcement (pp 31-60). New York: Russell Sage Foundation.

Greshman, F.M. \& Elliott, S.N. (2007). Social Skills Rating System. Toronto: Pearson Publishing.

Goldberg, J. S., \& Carlson, M. J. (2014). Parents' relationship quality and children's behavior in stable married and cohabiting families. Journal of Marriage and Family, 76(4), 762-777.

Goldberg, J. S., \& Carlson, M. J. (2015). Patterns and predictors of coparenting after unmarried parents part. Journal of Family Psychology, 29(3), 416-426.

Gonzalez, M., Jones, D., \& Parent, J. (2014). Coparenting experiences in African American families: An examination of single mothers and their nonmarital coparents. Family Process, 53(1), 33-54.

Grych J.H., \& Fincham F.D. (1990). Marital conflict and children's adjustment: a cognitive-contextual framework. Psychological Bulletin, 108(2), 267-90.

Grych, J. H., \& Fincham, F. D. (2001). Interparental conflict and child development: Theory, research and applications. Cambridge: Cambridge University Press. 
Grych, J., Oxtoby, C., Lynn, M. (2013). The effects of interparental conflict on children. In M.A. Fine \& F.D. Fincham, (Eds.). Handbook of family theories: A contentbased approach (pp.338-355). New York: Routledge.

Grych, J. H., Raynor, S. R., \& Fosco, G. M. (2004). Family processes that shape the impact of interparental conflict on adolescents. Development and Psychopathology, 16(3), 649-665.

Hetherington, E. M. \& Kelly, J. (2002). For better or worse: Divorce reconsidered. New York: Norton.

Hutchison, E.D. (2015). Dimensions of human behavior: The changing life course (5 $5^{\text {th }}$ ed.). Los Angeles, CA: Sage.

Jones, D. J. \& Lindahl, K.M. (2011). Coparenting in extended kinship systems: African American, Hispanic, Asian heritage, and Native American families. In J. P. McHale \& K. M. Lindahl (Eds.), Coparenting: A conceptual and clinical examination of family systems (pp. 61-79). Washington, DC, US: American Psychological Association.

Kamp Dush, C. M., Kotila, L. E., \& Schoppe-Sullivan, S. J. (2011). Predictors of Supportive Coparenting After Relationship Dissolution Among At-Risk Parents. Journal of Family Psychology, 25(3), 356-365.

Karberg, E. \& Cabrera, N. (2017). Family change and co-parenting in resident couples and children's behavioral problems, Journal of Family Studies, DOI: $\underline{10.1080 / 13229400.2017 .1367714 .}$

Kelly, J.B. \& Emery, R.E. (2003). Children's adjustment following divorce: Risk and resilience perspectives. Family Relations, 52, 352-362. 
Kerlinger, F., \& Lee, H. (2000). Foundations of Behavioral Research (4th ed.). Fort Worth, TX: Harcourt College.

Key Findings for Policy Makers. (2016, May 16). Retrieved from: http://www.fragilefamilies.princeton.edu/news/key-findings-policy-makers.

Kochhar, R. \& Cilluffo, A. (2017, November 1). How wealth inequality has changed in the U.S. since the Great Recession, by race, ethnicity and income. Retrieved from http://pewrsr.ch/2iSfbOS.

Kuersten-Hogan, R. (2017). Bridging the gap across the transition to coparenthood: Triadic interactions and coparenting representations from pregnancy through 12 months postpartum. Frontiers in Psychology, 8, 475.

Leinonen, J. A., Solantus, T. S., \& Punamäki, R. (2002). The specific mediating paths between economic hardship and the quality of parenting. International Journal of Behavioral Development, 26(5), 423-435.

Linares, L. O., Rhodes, J., \& Montalto, D. (2010). Perceptions of coparenting in foster care. Family Process, 49(4), 530-542.

Lindahl, K. M., \& Malik, N. M. (1999). Marital conflict, family processes, and boys' externalizing behavior in Hispanic American and European American Families. Journal of Clinical Child Psychology, 28(1), 12-24.

Manning, W. D., Brown, S. L., \& Stykes, J. B. (2014). Family complexity among children in the United States. Annals of the American Academy of Political and Social Science, 654(1), 48-65. 
Marchand-Reilly, J. F., \& Yaure, R. G. (2019). The Role of Parents' Relationship Quality in Children's Behavior Problems. Journal of Child \& Family Studies, 28(8), 2199-2208.

Margolin, G., Gordis, E. B., \& John, R. S. (2001). Coparenting: A link between marital conflict and parenting in two-parent families. Journal of Family Psychology, 15, 3-21.

Martin, A., Ryan, R. M., Riina, E. M., \& Brooks-Gunn, J. (2017). Coresidential father transitions and biological parents' coparenting quality in early and middle childhood. Journal of Family Issues, 38(5), 677-699.

McConnell, M. C., \& Kerig, P. K. (2002). Assessing coparenting in families of schoolage children: Validation of the Coparenting and Family Rating System. Canadian Journal of Behavioural Science, 34(1), 44.

McDaniel, B. T., Teti, D. M., \& Feinberg, M. E. (2017). Assessing coparenting relationships in daily life: The Daily Coparenting Scale (D-Cop). Journal of Child and Family studies, 26(9), 2396-2411.

McHale, J. P. (1995). Coparenting and triadic interactions during infancy: The roles of marital distress and child gender. Developmental Psychology, 31(6), 985.

McHale, J. P. (2007). When infants grow up in multiperson relationship systems. Infant Mental Health Journal, 28(4), 370-392.

McHale, J. P., (2011). Assessing Coparenting. In J.P. McHale, K.M. Lindahl, (Eds.), Coparenting: A conceptual and clinical examination of family systems (pp. 149 170). Washington, DC, US: American Psychological Association. 
McHale, J. P., Kuersten-Hogan, R., \& Rao, N. (2004). Growing points for coparenting theory and research. Journal of Adult Development, 11(3), 221-234.

McHale, J.P., Lauretti, A., Talbot, J.A., \& Pouquette, C. (2002). Retrospect and prospect in the psychological study of coparenting. In J.P. McHale \& W. Grolnick (Eds.), Retrospect and prospect in the psychological study of families (pp. 127-165).

Mahwah, NJ: Lawrence Erlbaum.

McHale, J.P. \& Lindahl, K.M. (2011). Introduction: What is coparenting? In J.P. McHale, K. M. Lindahl, (Eds.), Coparenting: A conceptual and clinical examination of family systems (pp. 3-12). Washington, DC, US: American Psychological Association.

McLanahan, S. (2009). Fragile families and the reproduction of poverty. The Annals of the American Academy of Political and Social Science, 621(1), 111-131.

McLanahan, S., \& Beck, A. (2010). Parental relationships in fragile families. Future of Children, 20(2), 17-37.

McLanahan, S. \& Carlson, M. (2004). Fathers in fragile families. In Lamb (Ed.). The Role of the Father in Child Development (4th ed.) (pp. 368 - 396). Hoboken, N.J.: Wiley.

McLanahan, S., Garfinkel, I., Reichman, N., Teitler, J., Carlson, M., \& Audigier, C. N. (2003). The fragile families and child wellbeing study: Baseline national report. Princeton, NJ: Center for Research on Child Wellbeing, Princeton University.

McLanahan, S., Tach, L., \& Schneider, D. (2013). The causal effects of father absence. Annual Review of Sociology, 39, 399-427. 
Meyer, D. R., \& Carlson, M. J. (2014). Family complexity: Implications for policy and research. Annals of the American Academy of Political and Social Science, 654(1), 259-276.

Mincy, R.B. and H. Pouncy. (2007). "Baby Fathers and American Family Formation.” Essay, Future of the Black Family Series. Center for Marriage and Families at the Institute for American Values, New York.

Minuchin, P. (1985). Families and individual development: Provocations from the field of family therapy. Child Development, 56(2), 289-302.

Minuchin, S. (1974). Families and family therapy. Cambridge, MA: Harvard University Press.

Mistry, R. S., Lowe, E. D., Benner, A. D., \& Chien, N. (2008). Expanding the family economic stress model: Insights from a mixed-methods approach. Journal of Marriage and Family, 70(1), 196-209.

Mistry, R. S., Vandewater, E. A., Huston, A. C., \& McLoyd, V. C. (2002). Economic well-being and children's social adjustment: The role of family process in an ethnically diverse low-income sample. Child Development, 73(3), 935-951.

Montalto, D. \& Linares, L.O. (2011). Coparenting practices among families in the foster care system. In J. P. McHale, K. M. Lindahl, (Eds.), Coparenting: A conceptual and clinical examination of family systems (pp. 251-268). Washington, DC, US: American Psychological Association.

Office of Child Support Enforcement: Annual Report to Congress FY 2016 (2020, Feb 2). Retrieved from: 
https://www.acf.hhs.gov/sites/default/files/programs/css/fy_2016_annual_report.p df.

Osborne, C., \& McLanahan, S. (2007). Partnership instability and child wellbeing. Journal of Marriage and Family, 69(4), 1065-1083.

Panter-Brick, C., Burgess, A., Eggerman, M., McAllister, F., Pruett, K., \& Leckman, J. F. (2014). Practitioner review: Engaging fathers-Recommendations for a game change in parenting interventions based on a systematic review of the global evidence. Journal of Child Psychology and Psychiatry, 55(11), 1187-1212.

Patterson, C.J. \& Farr, R.H. (2011). Coparenting among lesbian and gay couples. In J. P. McHale, K. M. Lindahl, (Eds.), Coparenting: A conceptual and clinical examination of family systems (pp. 127-146). Washington, DC, US: American Psychological Association.

Perry, A. R., Harmon, D. K., \& Leeper, J. (2012). Resident Black fathers' involvement: A comparative analysis of married and unwed, cohabitating fathers. Journal of Family Issues, 33(6), 695-714.

Petren, R.E., Ferraro, A.J., Davis. T.R. \& Pasley, K. (2017). Factors linked with coparenting support and conflict after divorce. Journal of Divorce \& Remarriage, 58(3), 145-160.

Pew Research Center. (Ret: 2015 December 17). Parenting in America: Outlook, worries, aspirations are strongly linked to financial situation. Retrieved from: http://www.pewsocialtrends.org/files/2015/12/2015-12-17_parenting-inamerica_FINAL.pdf. 
Randles, J. (2019). The means to and meaning of "being there" in responsible fatherhood programming with low-income fathers. Family Relations: An Interdisciplinary Journal of Applied Family Studies, 69(1), 7-20.

Reichman, N., Teitler, J., Garfinkel, I., McLanahan, S. (2001). Fragile families: Sample and design. Child and Youth Services Review, 23(4/5), 303-326.

Roopnarine, J.L. (2004). African American and African Caribbean fathers: Level, quality, and meaning of involvement. In Lamb (Ed.). The role of the father in child development (4th ed.) (pp. 368-396). Hoboken, N.J.: Wiley.

Royse, D. (2011). Research Methods in Social Work (6 ${ }^{\text {th }}$ Ed). Cengage Learning: Belmont, CA.

Sawhill, I. (2014). Family complexity: Is it a problem, and if so, what should we do? Annals of The American Academy of Political and Social Science, 654(1), 240-244.

Saxbe, D., Rodriguez, A., Margolin, G. (2013). Understanding conflict in families: Theoretical frameworks and future directions. In M.A. Fine \& F.D. Fincham, (Eds.). Handbook of family theories: A content-based approach (pp.169-189). New York: Routledge.

Schoppe-Sullivan, S.J., Altenburger, L.E, Lee, M.A., Bower, D.J., Kamp Dush, C.M.(2015). Who are the gatekeepers? Predictors of maternal gatekeeping. Parenting, 15(3), 166-186.

Setia M. S. (2016). Methodology Series Module 3: Cross-sectional Studies. Indian Journal of Dermatology, 61(3), 261-264. 
Shannon, J. D., Cabrera, N. J., Tamis-LeMonda, C., \& Lamb, M. E. (2009). Who stays and who leaves? Father accessibility across children's first 5 years. Parenting: Science and Practice, 9(1-2), 78-100.

Skinner, E. A., \& Wellborn, J. G. (1997). Children's coping in the academic domain. In S.Wolchik \& I.N. Sandler (Eds.). Handbook of children's coping (pp. 387422). Springer, Boston, MA.

Stright, A. D., \& Neitzel, C. (2003). Beyond parenting: Coparenting and children's classroom adjustment. International Journal of Behavioral Development, 27, 3140.

Sturge-Apple, M. L., Davies, P. T., \& Cummings, E. M. (2010). Typologies of family functioning and children's adjustment during the early school years. Child development, 81(4), 1320-1335.

Sturge-Apple, M. L., Davies, P. T., Winter, M. A., Cummings, E. M., \& Schermerhorn, A. (2008). Interparental conflict and children's school adjustment: The explanatory role of children's internal representations of interparental and parentchild relationships. Developmental Psychology, 44(6), 1678-1690.

Sznitman, A.G., Van Petegem, S., \& Zimmermann, G. (2018). Exposing the role of coparenting and parenting for adolescent personal identity processes. Journal of Social and Personal Relationships. Retrieved from: https://doi.org/10.1177/0265407518757707.

Tabachnick, B. G. and Fidell, L.S. (2013). Using Multivariate Statistics (6th ed.) Boston, MA: Pearson. 
Teubert, D., \& Pinquart, M. (2010). The association between coparenting and child adjustment: A meta-analysis. Parenting: Science and Practice, 10(4), 286-307.

Umemura, T., Christopher, C., Mann, T., Jacobvitz, D., \& Hazen, N. (2015). Coparenting problems with toddlers predict children's symptoms of psychological problems at age 7. Child Psychiatry and Human Development, 46(6), 981-996.

United States Census Bureau (2019). How the Census Bureau Measures Poverty. Retrieved from: https://www.census.gov/topics/incomepoverty/poverty/guidance/poverty-measures.html.

Van Egeren, L. A. (2003). Prebirth predictors of coparenting experiences in early infancy. Infant Mental Health Journal, 24(3), 278-295.

Waller, M. R. (2012). Cooperation, conflict, or disengagement? Coparenting styles and father involvement in fragile families. Family Process, 51(3), 325-342.

Wallerstein, J.S. \& Kelly, J.B. (1980). Surviving the breakup: How parents and children cope with divorce. New York: Basic Books.

What Does Cronbach's Alpha Mean? (n.d.). UCLA: Statistical Consulting Group. Retrieved from: https://stats.idre.ucla.edu/spss/faq/what-does-cronbachs-alphamean/.

Woodcock, R.W., McGrew, K.S., \& Mather, N. (2001). Woodcock-Johnson III Tests of Achievement. Itasca, IL: Riverside Publishing.

Zhang, X. (2014). Family income, parental education and internalizing and externalizing psychopathology among 2-3-year-old Chinese children: The mediator effect of parent-child conflict. International Journal of Psychology, 49(1), 30-37. 


\section{CURRICULUM VITA \\ Shannon M. Mount}

\section{EDUCATION}

\begin{tabular}{ll}
\hline Ph.D. & University of Louisville - Louisville, KY \\
& Doctor of Philosophy in Social Work, May 2020 \\
& Dissertation Title: Coparenting and School Success: Perspectives of \\
& Fathers \\
& Dissertation Award: Graduate Dean's Citation Award \\
& Advisor: Dr. Armon Perry \\
& Indiana University -- Indianapolis, IN \\
M.S.W. & Master of Social Work Degree, May 1995 \\
& Grade Point Average 3.92/4.0 \\
& Indiana University -- New Albany, IN \\
B.A. & Bachelor of Arts Degree in Psychology, May 1993 \\
& Grade Point Average 3.85/4.0 with High Distinction
\end{tabular}

\section{PROFESSIONAL LICENSES \& CERTIFICATES}

\begin{tabular}{lll}
\hline $2013-$ Present & Registered Family Relations Mediator & Indiana Continuing Legal \\
Education & & Indiana Professional \\
$\begin{array}{l}1999-\text { Present } \\
\text { Licensing Agency } \\
1998-\text { Present } \quad \text { Licensed School Social Worker }\end{array}$ & Indiana Department of \\
Education &
\end{tabular}

\section{ACADEMIC \& PROFESSIONAL EXPERIENCE}

SCOTT COUNTY SCHOOL DISTRICT 2 -- Scottsburg, IN

Director of Counseling Services

High School College \& Career Counselor/Social Worker

August 2013 - Present

- Currently overseeing \$350,000/4-year grant project. Lilly Comprehensive Counseling Initiative: https://lillyendowment.org/our-work/education/indiana-k12/comprehensive-counseling-initiative/ 
- Oversee districts counseling services and supervise district school counselors/social workers

- Provide a comprehensive counseling plan for college \& career development for all K-12 students

- Oversee $21^{\text {st }}$ Century Scholars program (for low-income and first generation college students)

- Write grants for the school corporation

- Manage the dual credit program and serve as a liaison between the high school and the partner colleges Elementary School Counselor/Social Worker January 2000 - June 2013

- Provide school counseling services to approximately 500-800 students

- Provide individual and small group counseling

- Develop and conduct classroom guidance lessons

- Provide consultation to classroom teachers and parents

- Serve as a member of the Student Support Team (Response to Intervention model); School Safety Committee (includes work on discipline issues, bullying and overall school atmosphere); School Improvement Committee, High Ability District Committee

- Train and Implement Conflict Manager Program (grade 3-5)

CHILD \& FAMILY ADVOCATES, INC. - Scottsburg, IN Executive Director of non-profit organization May 2016 - Present

- Manage not-for-profit with the mission to educate families about the danger of family conflict

- Responsible for fund raising and grant writing

- Provide group and individual services to children and parents

- Provide family mediation services

IVY TECH COMMUNITY COLLEGE - Sellersburg, IN

Adjunct Instructor January 2017 - Present

- Teach in-person courses in Sociology and Human Services

- Use Canvas platform as instructional resource

INDIANA UNIVERSITY - New Albany, IN Adjunct Instructor August 2018 - Present

- Teach in-person courses in Sociology and Human Services

- Use Canvas platform as instructional resource

INDIANA PARENTING MATTERS - Scottsburg, IN Owner and LCSW Practitioner January 2012 - July 2017 
- Own and Manage Private Business specializing in intervening with families in conflict

- Write local and state grants to maintain the pro bono family counseling services provided to families in a four county region

- Provide family mediation as a Certified Family Domestic Relations Mediator (listed on Indiana Mediator Registry)

UNIVERSITY OF LOUISVILLE - Louisville, KY

Teaching Assistant/Research Assistant August 2014 - June 2016

- Team taught Human Behavior and Social Environment with tenured faculty

- Assist director of doctoral program with research tasks including qualitative interviews, training, and curricula development

SCOTT COUNTY OFFICE OF FAMILY AND CHILDREN -- Scottsburg, IN Privately Contracted Clinical Social Worker for Child Protective Services October 2000 - May 2005

- Provide direct home based clinical social work services for assigned cases with Child Protective Services

- Home based clinical social work services include individual and family counseling, crisis intervention, case management, court appearances, and advocacy

REASON FOR LEAVING: Contracted cases were closed.

JENNINGS COUNTY SCHOOL CORP. -- North Vernon, IN Elementary School Counselor/Social Worker August 1998 - December 1999

- Provided school counseling services to two elementary schools approximately 800 students

- Provided individual and small group counseling

- Developed and conducted classroom guidance lessons to grades K - 6

- Conducted assessments for students suspected of ADD/ADHD characteristics

- Provided consultation to classroom teachers and parents

- Served as a member of the Crisis Response Team

- Served as member of Building Based Support Team (BBST)

REASON FOR LEAVING: Accepted a position closer to home.

PLEASANT RUN, INC -- Indianapolis, IN Program Manager -- South Region April 1998 - August 1998

- Hired, evaluated, and supervised Master's level Social Workers

- Developed larger service area for Home Based Preservation Services

- Served as liaison between contract agents and Pleasant Run

REASON FOR LEAVING: Moved back to Scott County from Indianapolis. 


\section{PROFESSIONAL \& ACADEMIC CONFERENCE PRESENTATIONS}

Oakley-Jeppe, J. (College Board), Dorton, M (Purdue University), Thieme Arterberry, S (Indiana University), and Mount, S (Scott County School Dist 2) (Feb 2019) Partnering for Big Time Success. College Board Midwestern Regional Forum 2019, Cleveland, OH

Acosta, J (College Board), Dorton, M (Purdue University), Thieme Arterberry, S (Indiana University), and Mount, S (Scott County School Dist 2) (Feb 2019) Partnering for Big Time Success. The Indiana Association for College Admission Counseling, Indianapolis, IN

Sears, J.and Mount, S., (2017) Peer Mentors in Child Welfare: A Qualitative Study. Society for Social Work and Research, 21st Annual National Conference. New Orleans, LA (was not able to attend).

van Zyl, M., Verbist, A. N., Thomas, T.A., Sears, J., Wilfong, J., Mount, S., Sabree, R. (2016). Points of View in the Science of Social Work Debate. Society for Social Work and Research, $20^{\text {th }}$ Annual National Conference. Washington, DC.

Mount, S. (2015). Addressing Child and Adolescent Behavior Problems Using a Risk and Resiliency Framework. Victory Over Violence Conference. Louisville, KY.

Mount, S. (2005). Olweus Bullying Prevention Model: One School's Journey. Paul Munger Conference, Indianapolis, IN

\section{PUBLICATIONS}

Sears, J. S., Hall, M. T., Harris, L. M., Mount, S., Willauer, T., Posze, L., \& Smead, E. (2017). 'Like a marriage': Partnering with peer mentors in child welfare. Children And Youth Services Review, 7480-86. doi:10.1016/j.childyouth.2017.01.023

van Zyl, M., Verbist, A. N., Thomas, T.A., Sears, J., Wilfong, J., Mount, S., Sabree, R. (In press). Mapping the science of social work debate: An exercise in doctoral education. Journal of Social Work Education.

Wilfong, J., Toldi, A., Ball, D., Verbist, A.N., Sears, J., Mount, S., Fabian, G., Sabree, R., Schmidt, V., Lawson, T., Kiss, J., Soelter, E. (In revision). Social work student's perceptions of social work: A Cross country comparison.

\section{MEMBERSHIPS AND CERTIFICATIONS}

- National Association of Social Workers 
- Indiana Association of School Social Workers

- Licensed School Services Personnel -- School Social Worker

- Academy of Certified Social Workers

- State of Indiana Licensed Clinical Social Worker

- Certified Prenatal Care Coordinator

- Certified to conduct Myers Briggs Type Indicator

- Trainer "Bridges out of Poverty" (Payne, DeVol, Dreussi Smith)

- Trainer "Olweus Bullying Prevention Model" (Olweus)

- Certified Presenter "Guiding Good Choices" (Hawkins \& Catalano)

- Certified Presenter "LifeSkills" (Botvin)

\section{HONORS}

- 1996 New Hope Child and Family Services Leadership Award

- Psi Chi -- National Psychology Honor Society

- Alpha Delta Mu -- National Social Work Honor Society

- IUS \& IUPUI Graduated with High Distinction

- Recognized ASCA Model Program (RAMP) - American School Counselor Association

- Robert Wood Johnson, Center for Creative Leadership, Community Coalition Leadership Program, Cohort 2013

- UofL Graduate School Dean's Citation Ward for Dissertation 